\title{
El frente orogénico activo de los Andes centrales a la latitud de la ciudad de Mendoza (32 $\left.50^{\circ}-33^{\circ} \mathrm{S}\right)$
}

\author{
Stella M. Moreiras ${ }^{1}$, Laura B. Giambiagi ${ }^{1}$, Silvana Spagnotto ${ }^{2}$, Silvina Nacif ${ }^{3}$, José Mescua ${ }^{1}$, \\ Rafael Toural ${ }^{1}$
}

\author{
${ }^{I}$ CONICET-Instituto Argentino de Nivología, Glaciología y Ciencias Ambientales (IANIGLA)-Centro Científico Tecnológico (CCT). \\ Avda. Ruiz Leal s/n. Parque General San Martín. CP 5500, Mendoza, Argentina. \\ moreiras@mendoza-conicet.gob.ar; lgiambia@mendoza-conicet.gob.ar; jmescua@mendoza-conicet.gob.ar; raftoural@gmail.com \\ ${ }^{2}$ Universidad Nacional de San Luis, Ejército de los Andes 950, D5700HHW, San Luis, Argentina. \\ pampa113@gmail.com \\ ${ }^{3}$ Instituto Geofísico Sismológico Volponi, Universidad Nacional de San Juan, Meglioli 1160-S. 5400, Rivadavia, San Juan, Argentina. \\ nacif.silvina@gmail.com
}

\begin{abstract}
RESUMEN. El actual frente orogénico activo de los Andes centrales abarca parte del piedemonte mendocino, ubicado cerca de la ciudad capital, donde habitan al menos 800.000 personas. A pesar de ello, se carece de un estudio integral de fallas activas de este sector como potenciales fuentes sismogénicas. El presente estudio intenta soslayar esta falencia caracterizando las principales estructuras activas e integrándolas en un modelo cinemático-estructural elaborado a partir de tres perfiles estructurales balanceados. La deformación pedemontana se caracteriza por una población de fallas compresivas de rumbo N-S a NNE-SSW, cuyo nivel de despegue se ubicaría en el basamento pre-Silúrico, constituyendo un frente emergente de piel gruesa. La serie de corrimientos imbricados con despegue ubicado a 10-11 km de profundidad corresponde a las fallas Melocotón y Divisadero Largo, que presentan expresión superficial, y a una falla ciega denominada Maure, interpretada a partir del modelo estructural. Esta falla ciega transfiere su rechazo hacia un sistema de fallas retrovergentes de piel fina, con despegue dentro de los depósitos sinorogénicos neógenos, conformando de esta manera una zona de deformación triangular. Según los rasgos morfométricos de estos fallamientos, los sismos máximos probables rondan entre magnitudes de 5,4 y 6,8 Mw. Coexisten fallas transcurrentes sinestrales de rumbo NW-SE que se encuentran cortando a las fallas retrovergentes del sistema Cerro de la Gloria. Los resultados obtenidos fueron contrastados con datos de subsuelo y resolución de mecanismos focales de sismos locales superficiales.
\end{abstract}

Palabras clave: Neotectónica, Sismicidad superficial, Riesgo sísmico, Argentina.

\begin{abstract}
Current active front of the Central Andes at Mendoza city latitude $\left(3^{\circ}{ }^{\circ} \mathbf{5 0}-33^{\circ} \mathrm{S}\right)$. Current active front of the Central Andes comprises part of the western piedmont of the city of Mendoza, where more than 800,000 people are established. Despite this, a comprehensive study of active faults mainly as potential seismogenic sources is lacking in this crucial sector of the Andean foreland. The present study attempts to outwit this shortcoming by characterizing the main Quaternary structures and integrating them in a structural-kinematic model developed from three balanced structural cross-sections. The foreland deformation at the study area is characterized by a population of N-S to NNE striking reverse faults, whose detachment level will be located in the Pre Silurian basement, constituting a thick skin thrust front. The series of thrusts, rooted at a 10-11 km-depth detachment, corresponds to Melocotón and Divisadero Largo faults showing surficial expression, and a blind fault called Maure, inferred om the structural-kinematic model. The Maure blind fault transfers shortening to the west-vergent thin-skinned Cerro de la Gloria thrusts, thus forming a triangular deformation zone. According to morphometric features of these active faults, a probable maximum earthquake magnitude ranges from Mw 5.4 to 6.8. Furthermore, strike slip faults with NW-SE strike are cutting Cerro de la Gloria fault system. Results were contrasted with ground data and focal mechanisms of surficial local earthquakes.
\end{abstract}




\section{Introducción}

La historia sísmica de la región centro-oeste de Argentina es ampliamente conocida, ya que sismos de gran magnitud han sido reportados desde 1575 en la ciudad de Mendoza (Morey, 1938; Moreiras, 2004; Perucca y Moreiras, 2010). Esta actividad corresponde a sismos de intraplaca corticales someros ( $<30 \mathrm{~km}$ de profundidad), asociados a la transmisión de los esfuerzos compresivos generados a partir de la interacción de las placas Nazca y Sudamericana. Los eventos sísmicos se originan en el frente orogénico activo de los Andes centrales, que entre $\operatorname{los} 32^{\circ}$ y $33^{\circ} \mathrm{S}$ se encuentra en el piedemonte oriental de la precordillera (Bastías, 1986; Bastías et al., 1984, 1990, 1993; Cortés et al., 1999; Costa et al., 2000a). En este sector, el frente orogénico presenta un intenso neotectonismo que se evidencia por importante fallamiento de los depósitos cuaternarios, lo que indica que la actividad sísmica tuvo lugar en la región durante este período (Costa et al., 2000a, 2000b; Siame et al., 2006; Ahumada, 2006).

Estructuralmente, el frente orogénico activo está compuesto por una serie de fallas inversas con orientación meridional e inclinación tanto al este como al oeste. La mayor parte de estas fallas poseen registro de actividad holocénica (dentro de los últimos 10.000 años) (INPRES, 1985; Bastías et al., 1993; Costa et al., 2000a; Ahumada et al., 2006; Giambiagi y Moreiras, 2009; Moreiras, 2010; Frau et al., 20101). Si bien las estructuras con expresión superficial han permitido evaluar su potencial peligrosidad sobre la base de parámetros morfométricos, son escasos los estudios de detalle que permiten establecer el grado de actividad de dichas fallas. Se desconocen, además, las tasas de desplazamiento y los mecanismos focales de los sismos asociados a las mismas. Tampoco se ha analizado la posible presencia de fallas ciegas y la peligrosidad asociada a los fallamientos transversales que desplazan las estructuras principales. Esto limita la evaluación de la peligrosidad sísmica del área, lo cual es fundamental para un ordenamiento territorial adecuado y el establecimiento de los códigos de construcción sismorresistente que deben contemplarse para la construcción de infraestructuras en este sector.
El objetivo de este trabajo es describir el frente orogénico activo de los Andes centrales a la latitud de la ciudad de Mendoza entre los $32^{\circ} 50^{\prime}$ y $33^{\circ} \mathrm{S}$ (Fig. 1). Para ello se identificaron las potenciales fuentes sismogénicas de la región y se las integró en perfiles estructurales a fin de generar un modelo cinemático-estructural.

Una falla activa es aquella que presenta actividad sísmica histórica y/o que tiene una expectativa de ocurrencia en un intervalo de tiempo que afecte el desarrollo de la sociedad (Slemmons, 1977; Wallace, 1986) o que en opinión de expertos puede tener desplazamientos en el futuro. Nosotros utilizaremos este término para fallas con desplazamientos históricos o prehistóricos, ya que incluimos las estructuras que afectan niveles cuaternarios (1,8 Ma). Esto se basa fundamentalmente a un escaso registro histórico que rara vez excede los 500 años en esta porción de Sudamérica. En este caso podríamos considerar como equivalente al término falla con actividad cuaternaria (Costa et al., 2000a). La presencia de fallas activas en el piedemonte mendocino fue mencionada en forma temprana por varios autores (Ave Lallemant, 1892; Kurtz, 1897; Bodenbender, 1897, 1902; Keidel, 1907, 1910; Stappenbeck, 1910, 1913; Fossa Mancini, 1939). Las principales fallas longitudinales fueron analizadas mediante sus expresiones geomorfológicas por diferentes autores (Bastías et al., 1993; Vich y Pedrani, 1993; Mingorance, 2006). Además de estas estructuras, existen también fuentes potencialmente sismogénicas que no tienen expresión en el terreno denominadas fallas ciegas, cuya existencia solo es evidenciada mediante métodos indirectos como estudios geofísicos o modelado estructural. En este caso, la profundidad donde se encuentre la falla será fundamental para establecer los efectos superficiales de los sismos asociados.

\section{Marco tectónico y geológico}

El área de estudio se ubica en el dominio morfoestructural Cerrilladas Pedemontanas (Polanski, 1953; Regairaz y Zambrano, 1991). Este dominio está constituido por cerros de alturas inferiores a los $2.000 \mathrm{~m}$, separados por depresiones, los cuales pueden considerarse como una extensión hacia el este y hacia el sur de la precordillera Austral (Cortés et al., 2006). En este sector, la precordillera Austral

\footnotetext{
${ }^{1}$ Frau, C.; Gallucci, A.R.; Moreiras, S.M.; Giambiagi, L. 2010. Estudio de peligrosidad sísmica para el proyecto urbano Palmares Valley. Departamentos de Godoy Cruz y Las Heras, Provincia de Mendoza. Informe técnico No. 16/101 (Inédito). Centro Regional de Desarrollos Tecnológicos para la Construcción, Sismología y la Ingeniería Sísmica (CeReDeTeC). Facultad Regional Mendoza, Universidad Tecnológica Nacional: 82 p.
} 


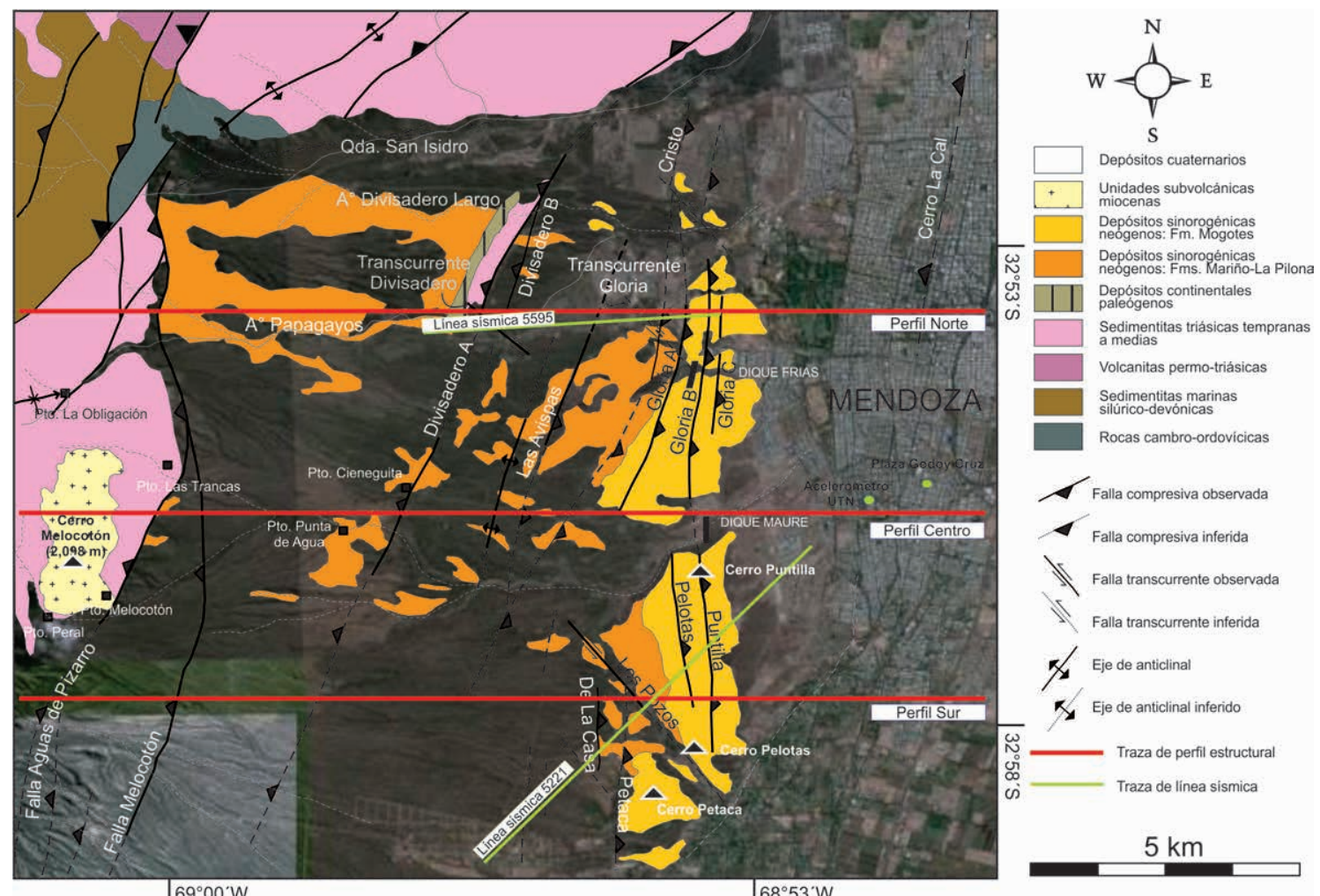

FIG. 1. Mapeo inicial de las fallas sismogénicas identificadas en la zona de estudio (Modificado de Frau et al., 2010¹).

corresponde a una faja plegada y corrida de piel gruesa bivergente, con un despegue profundo ubicado a los 10-11 km (Giambiagi et al., 2011). Las estructuras que comprenden esta faja pierden rechazo hacia el sur, donde comienzan a desarrollarse anticlinales desarrollados a partir de la inversión de las cuencas triásicas y de la generación de corrimientos de bajo ángulo que afectan a depósitos cuarternarios (Brooks et al., 2000; Chiaramonte et al., 2000; Vergés et al., 2007; Ahumada y Costa, 2009).

Una tasa de deformación regional de $\sim 3 \mathrm{~mm} /$ año se ha medido mediante GPS en este sector de la precordillera, pero se desconoce si esta deformación es uniforme en el retroarco, posiblemente concentrada en unos $50 \mathrm{~km}$ de ancho del frente orogénico, o bien si dicha deformación se está acumulando en alguna(s) estructura(s) específica(s) (Brooks et al., 2003; Kendrick et al., 2006; Schmidt et al., 2011).

Las unidades más antiguas aflorantes en el sector montañoso de precordillera están representadas por calizas cámbricas y pelitas ordovícicas (Fig. 1). Metasedimentitas siluro-devónicas, localmente in- truidas por plutones del Devónico temprano afloran discordantes sobre estas (Folguera et al., 2004). En fuerte discordancia angular se apoyan las sedimentitas marinas carboníferas superiores a pérmicas inferiores (Polanski, 1958). Por encima, las volcanitas permo-triásicas del Grupo Choiyoi se disponen con importante discordancia angular.

En el ámbito de piedemonte mendocino, propiamente dicho, las sedimentitas triásicas de la cuenca extensional Cuyana (Grupo Uspallata) constituyen las rocas más antiguas aflorantes. Por encima, se disponen depósitos jurásico-cretácicos de las formaciones Papagayos y Barrancas, paleógenos de la Formación Divisadero Largo, sinorogénicos mio-pliocenos de las formaciones Mariño, La Pilona y Río de los Pozos, y pliocenos superiores a pleistocenos tempranos de la Formación Mogotes (Irigoyen et al., 1999, 2000; Chiaramonte et al., 2000). Cubren a estas unidades en forma discordante y casi continua, niveles aluviales aterrazados asignados a las formaciones Mesones e La Invernada del Pleistoceno Inferior a Medio (Polanski, 1963), constituidas 
por gravas polimícticas poco consolidadas. Estos niveles se encuentran fuertemente disectados y se interdigitan con los depósitos del cono aluvial de río Mendoza hacia el oriente (Sepúlveda y López, 1998). Se diferencian depósitos limosos holocénicos donde se intercala un nivel cinerítico correlacionable con los depósitos de abanico distal del Borbollón donde cenizas volcánicas han sido datadas entre 16 y 27 ka (Olgiati y Ramos, 2003). En tanto, un nivel de ceniza volcánica denominada 'Pumicita Abrasiva' que aflora en este mismo sector fue datada 6.000 AC (Olgiati, 2002).

\section{Sismicidad histórica}

El frente orogénico andino entre las latitudes $30^{\circ}$ y $33^{\circ} \mathrm{S}$ presenta registros de actividad sísmica histórica (Morey, 1938; Bastías et al., 1993; INPRES, 1995; González et al., 2002; Moreiras, 2010) (Fig. 2). Al menos 7 sismos históricos corticales de magnitudes mayores a 7 han sido registrados en este segmento. El sismo del 15 enero 1944 (La Laja, Albardón) de Ms=7,4 (intensidad IX, San Juan) tuvo una profundidad de $11 \mathrm{~km}$ según Alvarado et al. (2005) y de $25 \mathrm{~km}$ de acuerdo con Meigs y Nabelek (2010). Dicho evento generó un desplazamiento vertical de $22 \mathrm{~cm}$ y un desplazamiento horizontal de $25 \mathrm{~cm}$ (Harrington, 1944), resultando en 6.000-10.000 víctimas fatales y $80 \%$ de las viviendas destruidas. El terremoto de 1977 (Caucete, San Juan) de Ms=7,4, registró rupturas superficiales en la falla AmpacamaNiquizanga (Volponi et al., 1978; Bastías, 1986).

El mayor sismo histórico registrado en la ciudad de Mendoza ( $\mathrm{Ms}=7,2$-intensidad IX-X), ocurrido el 20 de marzo de 1861, se generó en este sector. Para el mismo se propuso una fuente sismogénica correspondiente a la reactivación del sector septentrional de la zona de la falla Cerro La Cal (Mignorance, 2006; Schmidt et al., 2011). Este sismo con hipocentro somero generó rupturas superficiales y procesos de licuación de suelos, resultando en 6.000 a 12.000 víctimas fatales, dependiendo de la fuente histórica que se consulte (Loos, 1907).

El sismo del 26 de enero de 1985 (Ms 5,9) que afectó la ciudad de Mendoza se asoció a una falla con vergencia este, ubicada al este del anticlinal Barrancas, localizado inmediatamente al sur de la zona de estudio (Chiaramonte et al., 2000; Brooks et al., 2000).

A pesar de la historia sísmica de Mendoza, no existe un estudio integral y multidisciplinario de las potenciales fuentes sismogénicas de la región. En el caso particular del segmento estudiado $\left(32^{\circ} 50^{\prime}-33^{\circ} \mathrm{S}\right)$, se carece de estimaciones de los rangos de desplazamiento de los sistemas de fallas involucradas, análisis de los mecanismos focales de los sismos, estudio en detalle de los depósitos cuaternarios afectados y un modelo estructural que resuelva el estilo de deformación del piedemonte mendocino.

\section{Metodología}

Se identificaron y caracterizaron las estructuras con evidencias de actividad cuaternaria del piedemonte mendocino entre los $32^{\circ} 50^{\prime}$ y $33^{\circ} \mathrm{S}$ mediante sensores remotos y observaciones de campo (Burbank y Anderson, 2001). Para cada estructura, se midieron los parámetros morfométricos como rumbo, manteo, dirección del vector de deslizamiento ('rake'), rechazo y características físicas de la zona de falla. En función de la longitud de rotura superficial (L) y el área de ruptura (RA), se establecieron las magnitudes Mw máximas probables de los potenciales sismos, utilizando las regresiones de Wells y Coppersmith (1984) (Tabla 1).

Esta información se complementó con un estudio sistemático de poblaciones de fallas menores y fracturas asociadas a fallas mayores, a partir de la observación de la geometría, orientación en el espacio y sentido de movimiento. Esto permitió comprender la cinemática de las fallas mayores identificadas en el terreno. Para ello se siguió la hipótesis cinemática de Marrett y Allmendinger (1990), Twiss y Unruh (1998) y Gapais et al. (2000) para determinar la orientación y la magnitud relativa de los ejes principales de velocidad de deformación obtenidos a partir de las concentraciones máximas de ejes de extensión (T) y de acortamiento (P) de un gran número de datos cinemáticos.

A partir de los datos relevados, se realizaron tres perfiles estructurales balanceados en sentido este-oeste: a. Perfil Norte (32 $53^{\prime}$ ): Arroyo FríasCerro de la Gloria-Reserva Divisadero-Ea. San Isidro; b. Perfil Central $\left(32^{\circ} 55^{\prime}\right.$ ): Arroyo Maure-Estancia Papagallos; c. Perfil Sur (3257'): Palmares-Estancia La Ciénaga-Punta de Agua-Estancia Melocotón. (Fig. 1). Los mismos fueron generados a partir de la utilización del software 2DMove, utilizando los datos de campo y la interpretación de líneas sísmicas obtenidas por YPF $(05221,05582,05571,05592$, 042019-05 y 05595). Estos perfiles balanceados fueron 

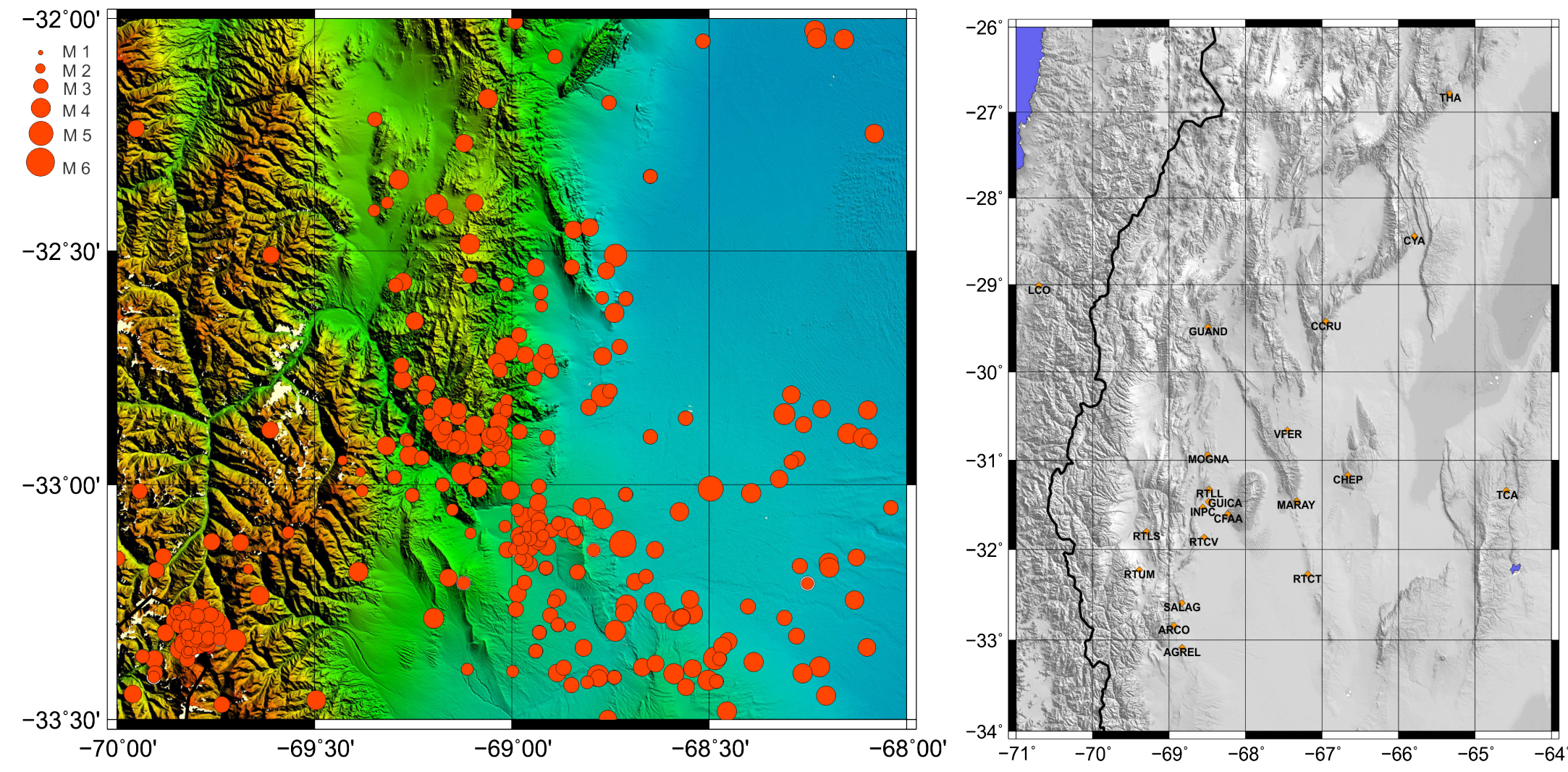

FIG. 2. Sismos de corteza de placa Sudamericana del catálogo de PDE (NEIC, 2014) entre 1999-2014 a partir de M>1, topografía Shuttle Radar Topography Mission y las estaciones sismológicas utilizadas para calcular los mecanismos focales. 
TABLA 1. PARÁMETROS MORFOMÉTRICOS DE LAS FALLAS PRINCIPALES.

\begin{tabular}{|c|c|c|c|c|c|c|c|c|c|c|c|c|c|c|c|c|c|}
\hline $\begin{array}{l}\text { Falla Cuaternaria } \\
\text { Piedemonte }\end{array}$ & $\begin{array}{c}\text { Edad } \\
\text { Último } \\
\text { mov. }\end{array}$ & Estilo & $\mathbf{R b}$ & $\mathbf{B z}$ & DZ & $\begin{array}{c}\mathbf{L} \\
\mathbf{k m}\end{array}$ & $\begin{array}{l}\mathbf{D} \\
\mathbf{m}\end{array}$ & $\begin{array}{r}\mathbf{R v} \\
\mathbf{m}\end{array}$ & $\begin{array}{c}\mathbf{R h} \\
\mathbf{m}\end{array}$ & $\begin{array}{l}\text { Ancho } \\
\text { m }\end{array}$ & $\begin{array}{c}\mathbf{W} \\
\mathbf{k m}\end{array}$ & $\begin{array}{c}\mathbf{R A} \\
\mathbf{K} \mathbf{m}^{2}\end{array}$ & $\begin{array}{l}\text { RD } \\
\text { mm/ } \\
\text { año }\end{array}$ & $\begin{array}{l}\mathrm{Re} \\
\mathrm{Ka}\end{array}$ & $\begin{array}{c}\text { Sismo } \\
\text { Mw (L) }\end{array}$ & $\begin{array}{l}\text { Sismo } \\
\text { Mw } \\
\text { (RA) }\end{array}$ & \\
\hline \multicolumn{18}{|l|}{ PERFIL NORTE } \\
\hline La Cal & Reciente & Inversa & 5 & $50 \mathrm{~W}$ & - & 30.7 & $16 / 3$ & - & - & - & - & - & $0,2-1$ & 1,3 & 6,8 & - & $\mathbf{L C}$ \\
\hline Falla Gloria A & Holocena & Inversa & 20 & $32 \mathrm{E}$ & - & $7 *$ & 3 & 2,1 & $2,14^{*}$ & 1,04 & - & - & - & - & - & - & \\
\hline Falla Gloria B & Holocena & Inversa & 33 & $31 \mathrm{E}$ & - & $15^{*}$ & 3,3 & 2,2 & $2,45^{*}$ & - & - & - & - & - & - & - & SFLG \\
\hline Falla Gloria C & Holocena & Inversa & 174 & $86 \mathrm{E}$ & $78 S$ & $5^{*}$ & - & - & - & 0,51 & - & - & - & - & - & - & \\
\hline Falla Gloria A Trans & Holocena & Rumbo & 6 & $73 E$ & - & $2 *$ & - & - & - & 0,3 & - & - & - & - & - & - & \\
\hline Falla Divisadero A & Pleist Sup & Inversa & 36 & $50^{\circ}$ & - & $13^{*}$ & $4,60^{*}$ & 3 & $3,57^{*}$ & 15 & 21 & 273 & $0,2-1$ & - & 6,37 & 6,45 & FDI \\
\hline Falla Divisadero B & Pleist Sup & Inversa & & & - & $3 *$ & - & - & - & - & - & - & - & - & - & - & $10 \mathrm{D}$ \\
\hline Falla Divisadero Largo Trans & Holocena & Rumbo & 126 & $89 W$ & $3 \mathbf{W}$ & - & - & 1,2 & - & 35 & - & - & - & - & - & - & \\
\hline \multicolumn{18}{|l|}{ PERFIL CENTRO } \\
\hline Falla Cristo & Holocena & Inversa & 31 & $53 \mathbf{E}$ & - & 7 & 0,6 & - & - & 4,96 & - & - & - & - & - & - & \\
\hline Falla Punta del Agua & Pleist Sup & Inversa & 44 & $77 \mathrm{E}$ & - & 7 & 0,42 & - & - & - & - & - & - & - & 6,41 & - & \\
\hline \multicolumn{18}{|l|}{ PERFIL SUR } \\
\hline Falla Puntilla-Gloria B sur & Holocena & Inversa & 35 & $47 E$ & - & $15^{*}$ & 3 & $2,04 *$ & $2,19^{*}$ & 2 & 4 & 60 & $0,2-1$ & 12,5 & 6,44 & 5,81 & \multirow{4}{*}{ SFLG } \\
\hline Falla Pelotas & Holocena & Inversa & 35 & $-\mathbf{E}$ & - & $3 *$ & - & - & - & - & 4 & 12 & - & - & 5,63 & 5,25 & \\
\hline Falla Petaca & Holocena & Inversa & 24 & $-W$ & - & $4 *$ & $3,05^{*}$ & 3 & $0,52 *$ & - & 4 & 16 & - & - & 5,77 & 5,02 & \\
\hline Falla Casa & Holocena & Inversa & 141 & $-\mathbf{E}$ & $85 N$ & $2 *$ & - & - & - & - & 4 & 8 & - & - & 5,43 & 4,95 & \\
\hline Falla Los Pozos Trans & Holocena & Rumbo & 110 & 69 & $6 N$ & 6 & - & - & - & - & - & - & - & - & - & - & \multirow{4}{*}{$\mathbf{F M}$} \\
\hline Falla Melocotón & Pleist Sup & Inversa & 2 & - & - & 9 & 2,6 & - & - & 4 & - & - & - & - & - & - & \\
\hline Falla Melocotón & Pleist Sup & Inversa & 2 & - & - & 40 & 2,6 & - & - & 4 & 19 & 760 & $0,2-1$ & 19 & 6,93 & 6,89 & \\
\hline Falla ciega Maure & - & - & - & - & - & 15 & - & - & - & - & 26 & 390 & - & - & 6,44 & 6,61 & \\
\hline
\end{tabular}

RB: Rumbo; Bz: Buzamiento; Dz: Deslizamiento ('rake'); Rv: Rechazo vertical; Rh: Rechazo horizontal; RA: Área de ruptura; RD: Rango desplazamiento (slip rate); Re: Recurrencia, datos en negrita: medidos; cursiva: trabajos previos; *: estimados; LC: Falla La Cal; SFLG: Sistema de fallas La Gloria; FDL: Falla de Divisadero Largo; FM: Falla el Maure. 
utilizados para el estudio cinemático-estructural. En el modelado se integraron los datos obtenidos del mapeo estructural de ubicación y disposición en el espacio de bancos y estructuras geológicas, datos de cinemática de las fallas, y contactos entre unidades.

Por otro lado, se recopilaron los sismos históricos de la región desde 1950 hasta la actualidad, tomados del Servicio Geológico de Estados Unidos (http:// earthquake.usgs.gov/earthquakes (última visita 22/03/2010)) [NEIC PDE catalog] y el Instituto Nacional de Prevención Sísmica (http://www.inpres.gov.ar (última visita 05/03/2013)). Utilizando datos de estaciones digitales de la red de INPRES (Instituto Nacional de Prevención Sísmica) y de redes globales a las cuales puede accederse desde la página web del consorcio IRIS (Incorporated Research Institutions for Seismology, www.iris. edu (última visita 22/03/2010)), se resolvieron los mecanismos focales para dos eventos sísmicos, uno ocurrido el 15 de septiembre de 2007 y el otro el 16 de octubre de 2008. Por otro lado, se analizaron los mecanismos focales de Nacif (2006), Alvarado et al. (2005) y el catálogo global Centroid Moment Tensor (CMT, http://www.globalcmt.org (última visita 27/03/2013)). Si bien no todos ellos corresponden al nivel de profundidad de las estructuras estudiadas y/o a la cinemática determinada para las fallas aflorantes, es de interés mostrarlos, ya que aportan a la información disponible de la zona.

En la resolución de mecanismos focales se usaron 14 estaciones de INPRES (RTUM, RTCV, RTLS, GUICA, INPC, CFAA, RTLL, RTCT, MOGNA, MARAY, VFER, CHEP, GUAND, CCRU, LCO y CYA) para el sismo del día 15/9/2007; mientras que para el sismo del 16/10/2008 se utilizaron 17 estaciones (AGREL, ARCO, SALAG, RTUM, RTCV, RTLS, INPC, CFAA, RTLL, MARAY, MOGNA, CHEP, VFER, GUAND, CCRU, TCA y THA) (Fig. 2). Las lecturas de los tiempos de arribo de las ondas $\mathrm{P}$ y S ('picking') fueron realizadas dentro del sistema SEISAN (Havskov y Ottemöller, 1999, 2003). Las velocidades de la onda $P$ usadas son $5,2 \mathrm{~km} / \mathrm{s}$ hasta los $45 \mathrm{~km}$ y $8,15 \mathrm{~km} / \mathrm{s}$ para profundidades mayores. Se obtuvo la localización y tiempo origen, utilizando el software HYPOCENTER (Lienert et al., 1995). Los mecanismos focales fueron resueltos con las primeras llegadas de ondas P, usando FOCMEC (Snoke et al., 1984), FPFIT (Reasenberg y Oppenheimer, 1985) y HASH (Hardebeck y Shearer, 2002, 2003). De las tres soluciones, preferimos las determinadas con
HASH porque, a diferencia de los otros métodos tiene en cuenta posibles errores en la localización del evento, incertezas inherentes al modelo de velocidad y errores en polaridad (Hardebeck y Shearer, 2003). Las soluciones de mecanismos focales se analizaron mediante la convención de Aki y Richards (1980), para rumbo/manteo/deslizamiento.

\section{Resultados}

\subsection{Fallas cuaternarias del sector de piedemonte}

Las principales fallas cuaternarias con expresión superficial que deforman el sector del piedemonte mendocino son de este a oeste: Cerro La Cal, Sistema de fallas Cerro de la Gloria y Cerro del Cristo, Punta del Agua, Divisadero Largo y Melocotón.

\subsubsection{Falla del Cerro La Cal}

La falla Cerro La Cal se ha caracterizado al norte de la ciudad de Mendoza y al parecer su traza continuaría hacia el sur ingresando por la calle Perú a la latitud de la zona de estudio. El desnivel topográfico de casi 1,2 m desarrollado a lo largo de esta arteria ha sido interpretado como la escarpa de la falla, la cual habría sido utilizada como terraplén para la localización del Ferrocarril Belgrano (INPRES, 1995). Es posible que esta falla presente una bifurcaron más occidental coincidente con la calle de Boulogne Sur Mer, aunque esto fue desestimado en el estudio de microsismidad desarrollado por el INPRES (1995).

Hacia el norte la traza de la falla es variable, con dirección principal N-S, y una longitud de 30,7 km (Mignonance, 2006). Su expresión boreal ha sido vinculada con el fallamiento Las Higueras (Ahumada y Costa, 2009). En superficie se manifiesta como una escarpa con frente hacia el este y manteo entre 40 y $50^{\circ}$ al oeste, en $16 \mathrm{~m}$ en niveles aluviales pleistocenos y en 2 a $3 \mathrm{~m}$ en depósitos modernos (INPRES, 1995). Por ende, la edad del último movimiento correspondería al Holoceno $(<15.000$ años). Mignorance (2006) establece deformación histórica para dos sectores de la falla, lo que habría generado dos escarpas de $3,5 \mathrm{~m}$ en total durante el sismo del año 1861. Salomon et al. (2013) realizaron una trinchera en una de estas escarpas, en la que han identificado al menos dos eventos sísmicos en los últimos 800 años, el último de los cuales correspondería al sismo de 1861. La tasa 
de movimiento para esta falla ha sido estimada en 0,57 a $0,77 \mathrm{~mm} /$ año (Mignorance, 2006) y 0,2-1,0 mm/año (INPRES, 1995; Costa et al., 2000a). En tanto Schmidt et al. (2011) establecieron una tasa de acortamiento variable en el tiempo, que habría sido de $0,9 \pm 0,3$ entre 12.100 y 3.900 años, de $1,5 \pm 0,3$ entre 3.900 y 770 años, y 5,4 $1,6 \mathrm{~mm} / \mathrm{a}$ entre 770 años y el presente. Esto señalaría una aceleración en el acortamiento que implica mayor actividad sísmica en la falla $\mathrm{La} \mathrm{Cal}$ en tiempos más recientes.

\subsubsection{Sistema de fallas Cerro de La Gloria y Cerro del Cristo}

El Sistema de fallas Cerro de La Gloria y Cerro del Cristo se expresa en lomadas pedemontanas alineadas en sentido N-S, constituidas por conglomerados plio-pleistocenos y bordeadas por depósitos de abanicos aluviales (Milana y Zambrano, 1996). Está integrado por varias fallas de trazos subparalelos N-S y NNE-SSW separados 500 a 1.000 m entre sí, con expresión superficial a lo largo de unos $15 \mathrm{~km}$. Son fallas inversas predominantemente de alto ángulo, aunque algunos trazos presentan ángulo bajo en superficie (Costa et al., 2000a). En campo se midieron manteos de $32^{\circ}$ a $86^{\circ}$ al este.

En la ladera sur del Cerro de la Gloria, en inmediaciones del anfiteatro, este sistema se expresa en una falla principal (A) con vergencia hacia el oeste $\left(21^{\circ}\right.$, $32^{\circ} \mathrm{E}$ ) que desplaza la Formación Mogotes al menos unos $3 \mathrm{~m}$ sobre limos holocenos que inclinan $12^{\circ}$ al oeste (Fig. 3). Una falla paralela a la principal (B) de idénticas características $\left(33^{\circ}, 31^{\circ} \mathrm{E}\right)$ afecta niveles de la Formación Mogotes un poco más al este. Los niveles pliocenos presentan una disposición casi vertical $\left(177^{\circ}, 84^{\circ} \mathrm{E}\right)$ (Fig. 4a) en este sector y son afectados por otra falla N-S subvertical (C) $\left(174^{\circ}\right.$, $86^{\circ} \mathrm{E}$ ) asociada a zonas de brechas y óxidos (Fig.4b) e interpretada como una estructura producida por cizalla interestratal.

En el sector sur del piedemonte (perfil sur), este sistema se manifiesta en dos trazas denominadas fallas Puntilla y Pelotas. La falla Puntilla $\left(35^{\circ}\right.$, $47^{\circ} \mathrm{E}$ ), cuya traza se alinea con las cimas de los cerros Puntilla y Pelotas, desplaza a la Formación Mogotes sobre limos cuaternarios. En tanto, los parámetros morfométricos de la falla Pelotas, la más occidental, no pudieron ser medidos. En este sector los niveles de la Formación Mogotes buzan $43^{\circ}$ hacia el este, verticalizándose en esa dirección hasta alcanzar ángulos de inclinación casi verticales, como se observó en el perfil norte del cerro de la Gloria (Fig. 4a). Niveles más occidentales de esta unidad medidos aguas arriba del dique Maure muestran manteos que varían entre $12^{\circ}$ y $21^{\circ}$ al este.

$\mathrm{Al}$ oeste de los cerros mencionados, en las inmediaciones del puesto de los Pozos, el sistema de fallas del Cerro de la Gloria afecta niveles neógenos de la Formación Mariño. Localmente esta falla se denomina Petaca al no poder continuar este lineamiento hacia el norte por falta de evidencias en terreno. La falla Petaca expone unos $3 \mathrm{~m}$ de depósitos aluviales jóvenes deformados $\left(24^{\circ}, 10^{\circ} \mathrm{W}\right)$. El rechazo preciso de esta falla no puede ser establecido debido a que se encuentra completamente cubierta. Hacia el oeste una falla paralela denominada la falla Casa pone en contacto niveles de la Formación Mariño, inclinando $39^{\circ} \mathrm{E}$, con niveles de dicha unidad que buzan $7^{\circ} \mathrm{E}$ en bloque oriental.

En el sector central de la zona de estudio, al oeste del dique Frías, se identificó el tramo medio de la falla

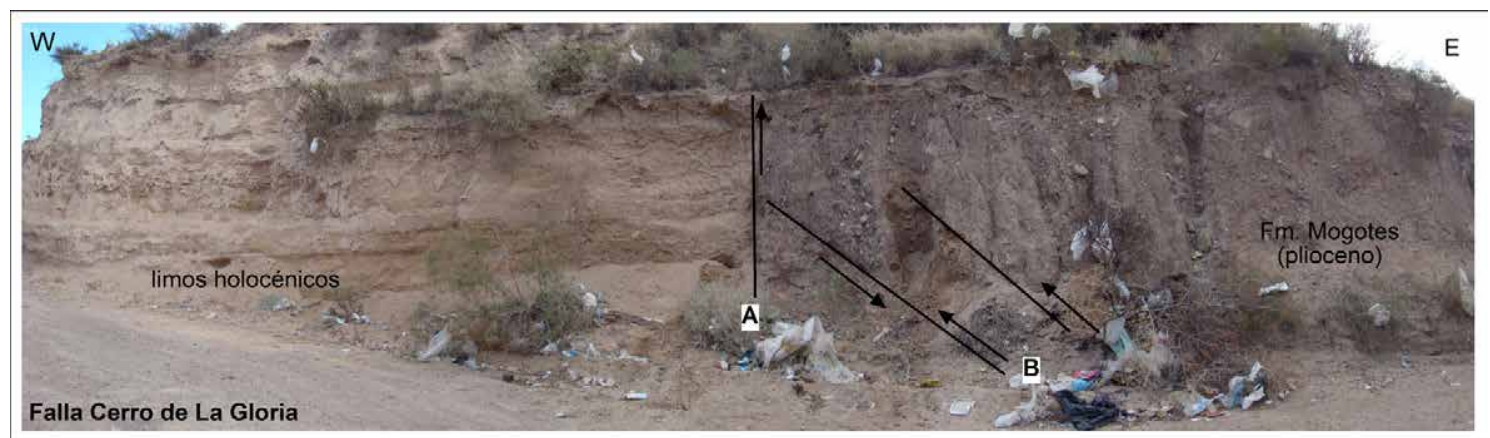

FIG. 3. Sistema de fallas Cerro de La Gloria: falla principal $\mathbf{A}\left(21^{\circ}, 32^{\circ} \mathrm{E}\right)$ que desplaza la Formación Mogotes unos $3 \mathrm{~m}$ sobre limos holocénicos buzantes $12^{\circ}$ al oeste y falla secundaria $\mathbf{B}\left(33^{\circ}, 31^{\circ} \mathrm{E}\right)$. 


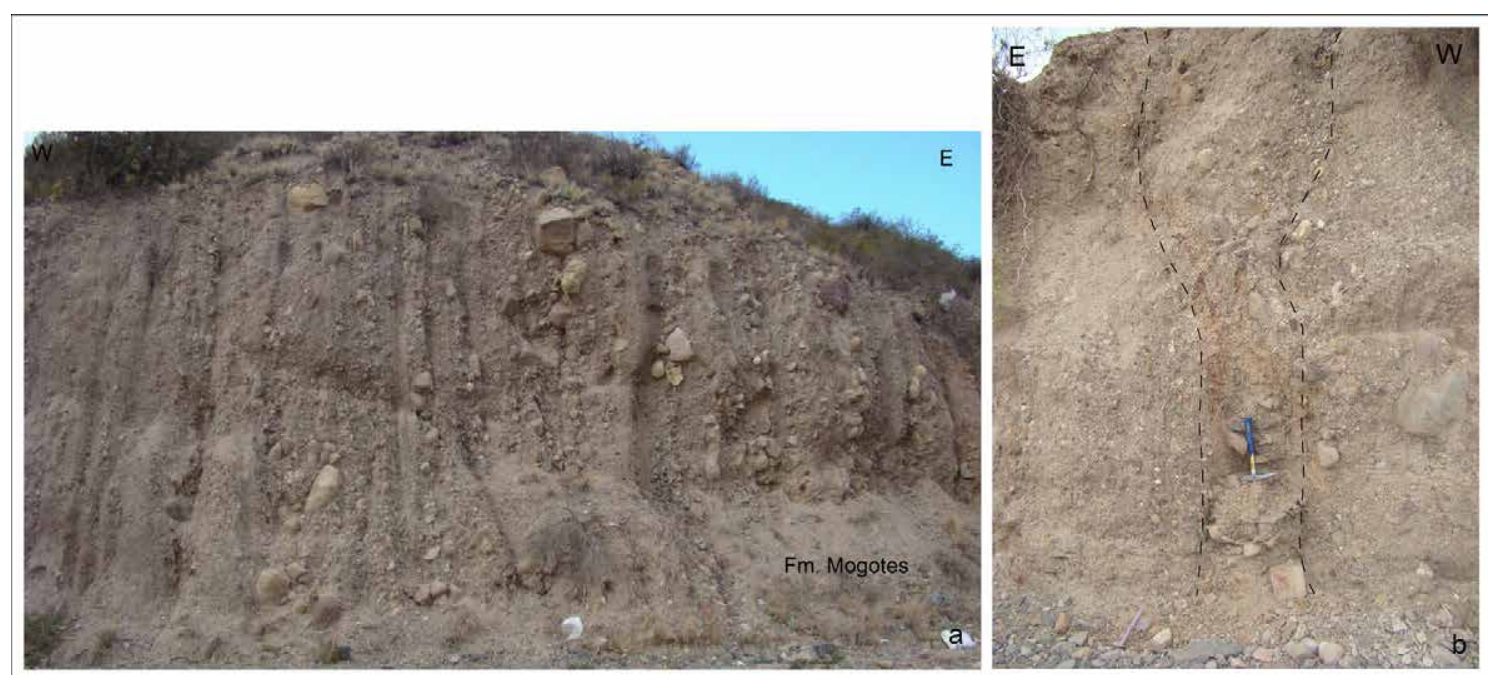

FIG. 4. a. Niveles pliocenos de la Formación Mogotes $\left(177^{\circ}, 84^{\circ} \mathrm{E}\right)$; b. Falla secundaria del sistema del Cerro de la Gloria (C) (174 , $86^{\circ} \mathrm{E}$ ) asociada a zonas de brechas y óxidos.

Cristo, afectando niveles de la Formación Mogotes. En esta sección la falla se manifiesta como una zona de falla de $5 \mathrm{~m}$ de ancho, asociada a fallas menores de rumbo NE-SW, con desplazamientos de 0,60 m. Esta falla puede reconocerse a lo largo de $7 \mathrm{~km}$.

\subsubsection{Falla Las Avispas}

La falla Las Avispas corresponde a una falla inversa con vergencia occidental y una inclinación de $40^{\circ}$ al este, que levanta los depósitos terciarios de la Formación Mariño sobre los sedimentos cuaternarios de la Formación Invernada (Vich y Pedrani, 1993). En el perfil central elaborado en este estudio, esta falla inversa de rumbo NE-SW presenta una mayor inclinación $\left(44^{\circ}, 77^{\circ} \mathrm{E}\right)$ y se encuentra afectando niveles terciarios de la Formación La Pilona.

\subsubsection{Falla Divisadero Largo}

La falla Divisadero Largo posee un rumbo aproximado norte-sur y atraviesa toda la zona de estudio. Sobre su traza se alinean los puestos de Las Higueritas, la Cieneguita y Agua de la Batea, alcanzando una longitud total aproximada de unos $13 \mathrm{~km}$.

La falla Divisadero Largo pierde rechazo hacia el sur. En la reserva Divisadero Largo, dicha estructura levanta rocas triásicas de la Formación Potrerillos por encima de sedimentos cuaternarios (Rodríguez y Barton, 1990), mientras que un poco más al sur pone en contacto rocas de la Formación Río Blanco sobre el mismo cuaternario. En el perfil del arroyo
Maure levanta a la Formación Mariño (Mioceno) sobre el cuaternario y hacia el sur afecta niveles pedemontanos de las Formaciones Los Mesones y La Invernada.

En el sector norte del piedemonte, en las inmediaciones de la Reserva Divisadero Largo, se observa que este sistema de fallas inversas ha sido afectado por un fallamiento transcurrente $\left(126^{\circ}, 89^{\circ} \mathrm{W}\right)$ (Fig. 5b) prácticamente vertical, el que habría generado un barreal de falla evidenciado por una secuencia de 0,70 m de espesor de limos finos (Fig. 5a). Esta falla transcurrente divide la falla Divisadero Largo en dos segmentos. El segmento meridional A ( $36^{\circ}$, $50^{\circ} \mathrm{W}$ ) corresponde a una zona de falla de unos $15 \mathrm{~m}$ de ancho (Fig. 6), mientras que la zona de falla del segmento $\mathrm{B}\left(120^{\circ}, 43^{\circ} \mathrm{W}\right)$ tiene un ancho de $20 \mathrm{~m}$.

\subsubsection{Falla Melocotón}

La falla Melocotón corresponde a un fallamiento inverso de rumbo norte-sur e inclinación al oeste (Bastías et al., 1993). Se expresa geomorfológicamente como una escarpa de falla de $9 \mathrm{~km}$ de largo en fanglomerados pleistocenos. A lo largo de su traza de aproximadamente $40 \mathrm{~km}$ de longitud, se alinean los puestos de El Peral, Las Trancas, Chambón y San Isidro. Estudios realizados en una trinchera revelan un desplazamiento de $2,6 \mathrm{~m}$ de material de basamento sobre limos cuaternarios no consolidados con contenido de gastrópodos datados $38 \mathrm{ka}$ (INPRES, 1995). Su tasa de movimiento se calcula en 0,2 a $1 \mathrm{~mm} / \mathrm{a}$ (Costa et al., 2000b). 


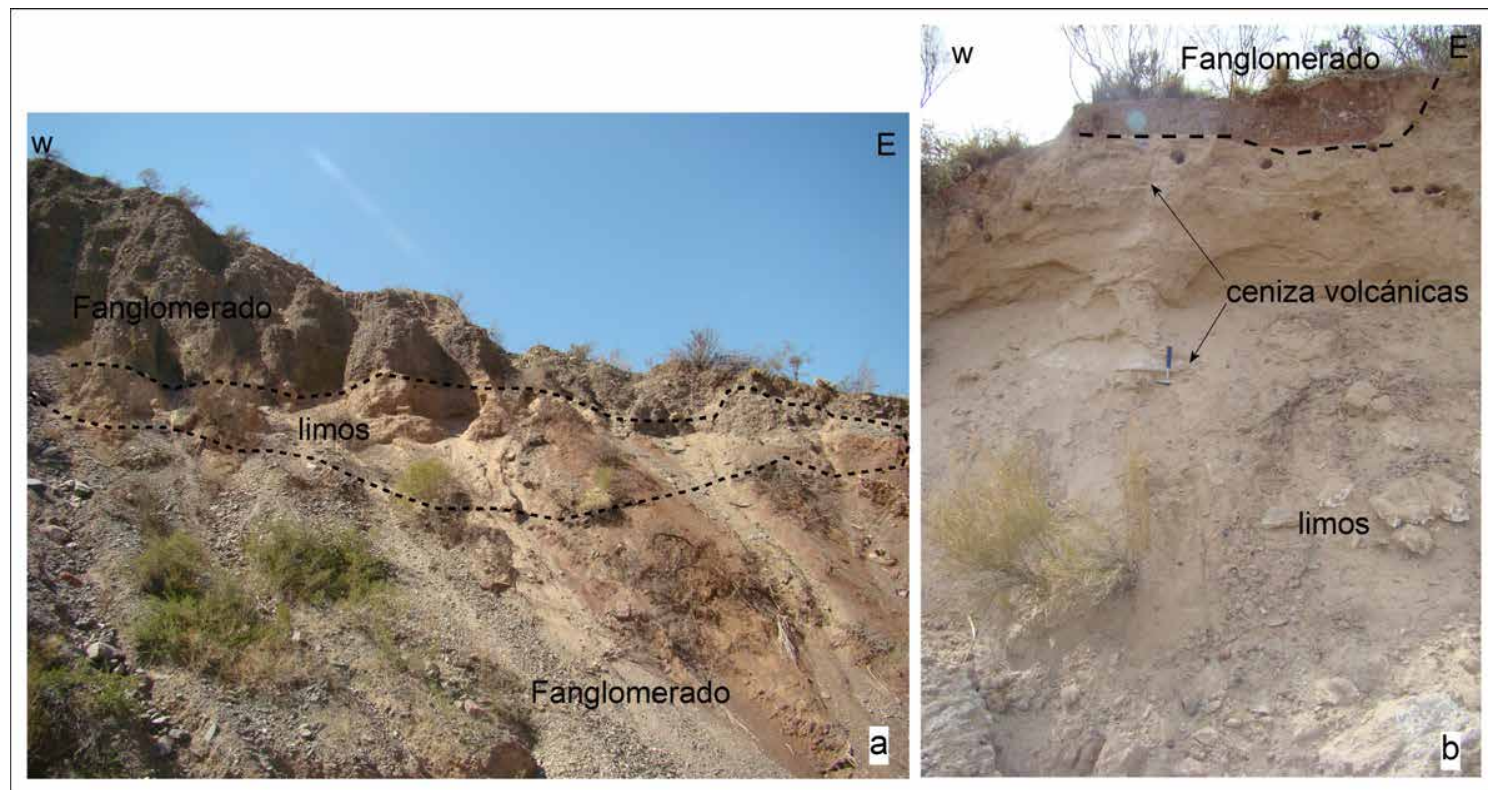

FIG. 5. a. Secuencia fina de $0,70 \mathrm{~m}$ de espesor posiblemente asociada a un barreal de falla ('sag ponds'), generado por la falla de transcurrente de Divisadero; b. Secuencia de $4 \mathrm{~m}$ de espesor de limos con niveles cineríticos en las inmediaciones del puesto Punta de Agua.

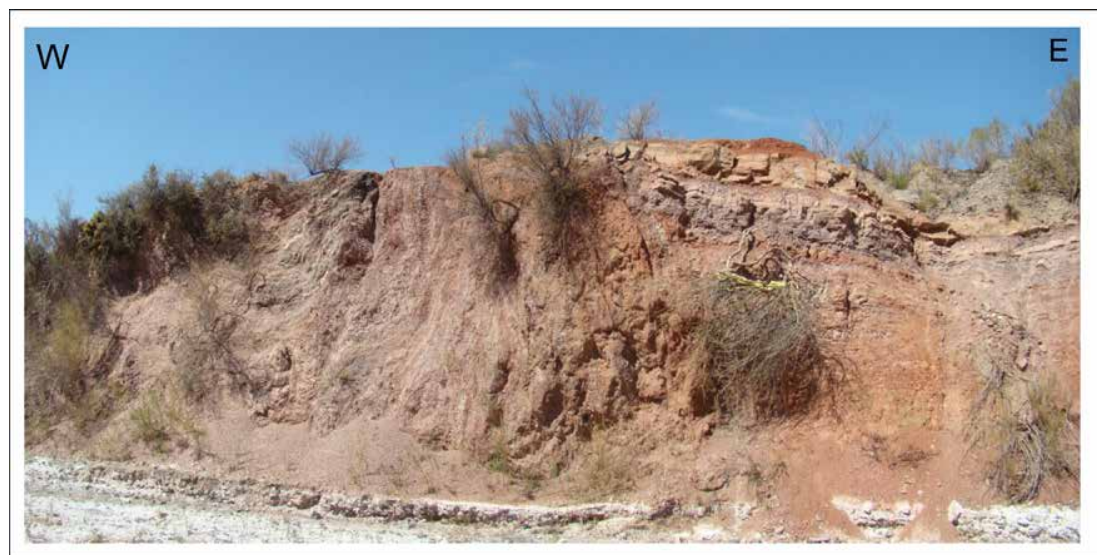

FIG. 6. Zona de falla del segmento A en donde se observan los niveles triásicos de la Formación Río Blanco completamente deformados.

\subsubsection{Fallas Transcurrentes}

Las fallas inversas del sistema del Cerro de la Gloria se encuentran cortadas por fallas transcurrentes. En el perfil sur se manifiesta la falla sinestral Pozos $\left(110^{\circ}, 69^{\circ} \mathrm{W}\right)$. Esta estructura corta todo el sistema de fallas norte-sur del sistema del Cerro de la Gloria, indicando un fallamiento holocénico más joven a los precedentes. Estudios geofísicos anteriores detectaron este fallamiento mediante sondeos eléctricos verticales al este del cerro de la Gloria (Perelló, 1991). Posiblemente estas fallas respondan a fallamientos más antiguos reactivados y sean los responsables de acomodar variaciones de desplazamiento entre las fallas inversas. Asimismo, tal como se ha adelantado, los segmentos A y B de la falla de Divisadero Largo son desplazados por un fallamiento transcurrente $\left(126^{\circ}, 89^{\circ} \mathrm{W}\right)$ descrito anteriormente (Fig. 7) (Tabla 1).

\subsection{Magnitudes máximas probables}

Las magnitudes máximas de sismos capaces de ser generados por las fallas cuaternarias analizadas 


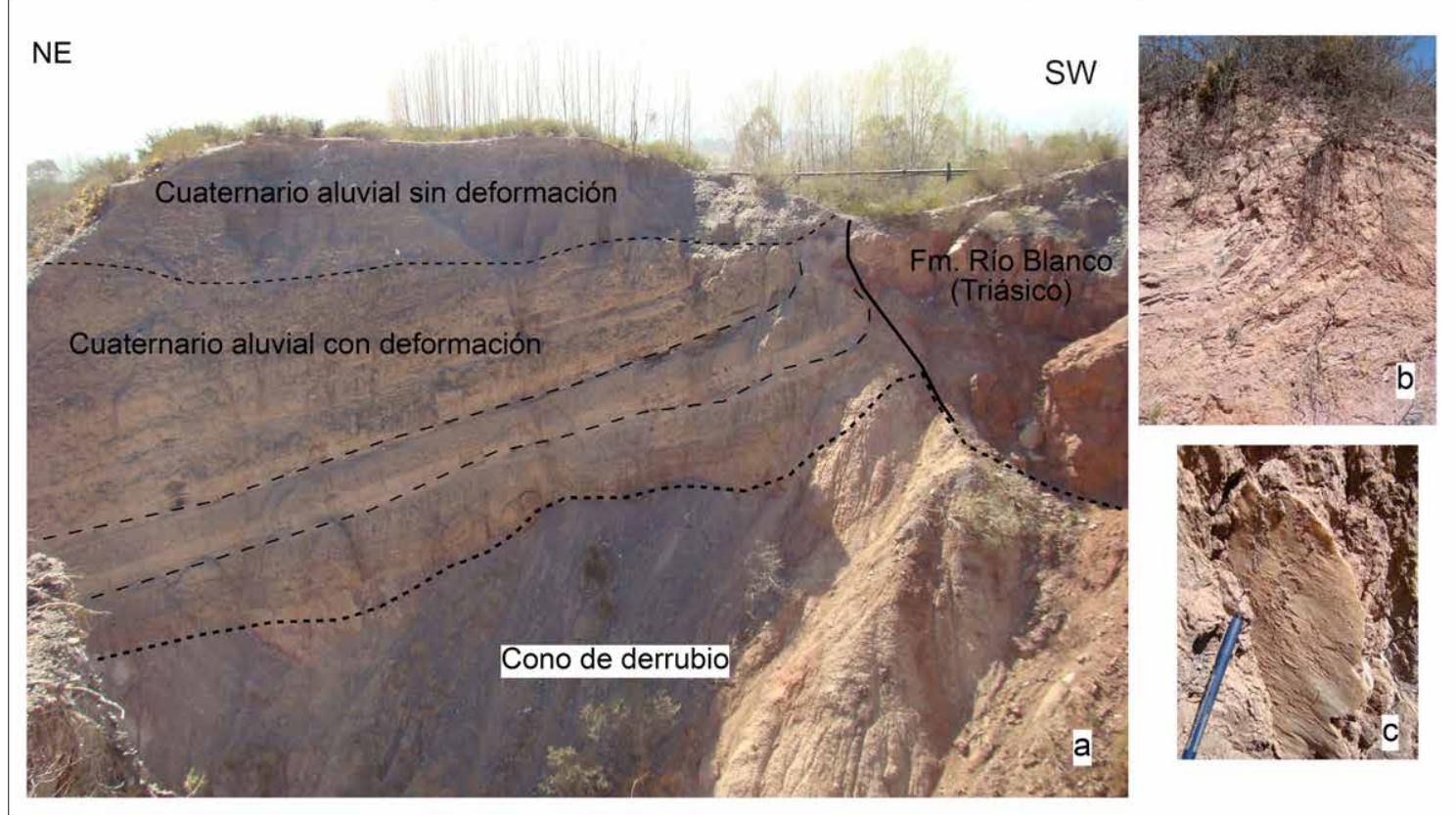

FIG. 7. a. Fallamiento transcurrente $\left(126^{\circ}, 89^{\circ} \mathrm{W}\right)$ que desplaza los segmentos A y B de la falla de Divisadero; $\mathbf{b}$. Zona de falla asociada; c. Estrías de fallas.

en el piedemonte mendocino fueron establecidas en función de la longitud de rotura superficial $(\mathbf{L})$ y el área de rotura de falla (RA) (Wells y Coppersmith, 1984) estimados en función del perfil sur balanceado elaborado. Los valores de magnitudes momento obtenidos fueron contrastados con los valores de desplazamiento medidos sobre las fallas (D) en terreno (Tabla 2).

En el caso de la falla La Cal, asignada como fuente sismogénica del sismo histórico de $1861(\mathrm{Ms}=7,2)$ (Mignorance, 2006), se establece una máxima probable $\mathrm{Mw}=6,8$ considerablemente inferior. Las fallas de Divisadero Largo y Cerro Melocotón presentarían magnitudes máximas de 6,45 y 6,9, respectivamente. En tanto el Sistema de fallas La Gloria se asocia a una máxima $\mathrm{Mw}=6,4$.

\subsection{Modelado cinemático-estructural}

La deformación pedemontana está caracterizada por una población de fallas compresivas y transcurrentes subordinadas, que constituyen el frente de deformación emergente de la precordillera como se describió en la sección anterior (Fig. 1). Las fallas compresivas corresponden a un sistema de fallas de rumbo N-S a NNE-SSW, cuyo nivel de despegue se ubicaría en el basamento pre-Silúrico, constituyendo un frente emergente de piel gruesa. El sector occidental del piedemonte posee fallas con vergencia oriental, mientras que el sector oriental del mismo corresponde a una zona de deformación triangular con fallas vergentes al oeste.

El modelo estructural/cinemático que mejor se ajusta a las estructuras observadas en superficie y a los datos cinemáticos de dichas estructuras, corresponde a una serie de corrimientos imbricados con despegue ubicado a 10-11 km de profundidad. Este despegue habría sido activo desde el Plioceno, durante la depositación de los conglomerados de la Formación Mogotes, hasta la actualidad. Los corrimientos principales corresponden a las fallas Melocotón y Divisadero Largo, que presentan expresión superficial, y a una falla ciega, interpretada a partir del modelo estructural, denominada falla Maure.

La falla Maure sería la responsable de la generación de la zona triangular asociada al sistema de fallas Cerro de la Gloria y Cerro del Cristo. El anticlinal ubicado inmediatamente al este de la falla Divisadero Largo, observado en los afloramientos de la Formación Mariño e interpretado en las líneas 
TABLA 2. SOLUCIONES DE MECANISMOS FOCALES DE LOS SISMOS ANALIZADOS PARA LAZONA DE ESTUDIO CON CONVENCIÓN DE AKI Y RICHARDS (1980) (FIG. 10).

\begin{tabular}{|c|c|c|c|c|c|c|c|c|c|c|c|}
\hline \multirow{2}{*}{ No. } & \multirow[b]{2}{*}{ día/mes/año } & \multirow{2}{*}{$\begin{array}{c}\text { Tiempo } \\
\text { Origen } \\
\text { hora:min:seg }\end{array}$} & \multirow{2}{*}{$\begin{array}{c}\text { Lat. } \\
\text { (grados) }\end{array}$} & \multirow{2}{*}{$\begin{array}{l}\text { Long. } \\
\text { (grados) }\end{array}$} & \multirow{2}{*}{$\begin{array}{l}\text { Prof. } \\
(\mathrm{km})\end{array}$} & \multirow{2}{*}{$\begin{array}{l}\text { Error } \\
\text { Prof. } \\
\\
(\mathbf{k m})\end{array}$} & \multicolumn{3}{|c|}{ Solución de Mecanismo Focal } & \multirow{2}{*}{ Mag. } & \multirow{2}{*}{ Fuente } \\
\hline & & & & & & & rumbo & manteo & Deslizamiento & & \\
\hline 1 & $07 / 05 / 2001$ & $05: 40: 06.10$ & $-33,021$ & $-69,080$ & 14,0 & $\leq 2,5$ & $\begin{array}{r}181,00 \\
15,00\end{array}$ & $\begin{array}{l}19,00 \\
71,00\end{array}$ & $\begin{array}{l}77,00 \\
94,00\end{array}$ & $\begin{array}{c}\mathrm{M}_{\mathrm{W}} \\
4,1\end{array}$ & $\begin{array}{l}\text { Alvarado } \\
\text { et al., } 2005\end{array}$ \\
\hline 2 & $05 / 12 / 2002$ & $21: 16: 24.9$ & $-32,90$ & $-69,07$ & 20,0 & $\pm 11,5$ & $\begin{array}{l}146,86 \\
278,89\end{array}$ & $\begin{array}{l}21,09 \\
75,52\end{array}$ & $\begin{array}{r}-44,01 \\
-105,50\end{array}$ & $\begin{array}{l}\mathrm{M}_{\mathrm{C}} \\
2,0\end{array}$ & Nacif, 2006 \\
\hline 3 & $21 / 12 / 2002$ & $09: 33: 44.4$ & $-32,99$ & $-69,05$ & 22,0 & $\pm 3,6$ & $\begin{array}{l}108,47 \\
273,18\end{array}$ & $\begin{array}{l}20,59 \\
70,08\end{array}$ & $\begin{array}{l}-75,65 \\
-95,32\end{array}$ & $\begin{array}{l}\mathrm{M}_{\mathrm{C}} \\
2,0\end{array}$ & Nacif, 2006 \\
\hline 4 & $23 / 08 / 2005$ & $19: 14: 9.10$ & $-32,930$ & $-69,140$ & 19,5 & $\pm 3,2$ & $\begin{array}{r}95,00 \\
346,00\end{array}$ & $\begin{array}{l}40,00 \\
75,00\end{array}$ & $\begin{array}{r}24,00 \\
127,00\end{array}$ & $\begin{array}{c}\mathrm{M}_{\mathrm{W}} \\
4,8\end{array}$ & CMT \\
\hline 5 & $15 / 09 / 2007$ & $19: 16: 36.8$ & $-32,87$ & $-68,96$ & 10,1 & $\pm 8,5$ & $\begin{array}{r}115,40 \\
9,90\end{array}$ & $\begin{array}{l}40,20 \\
77,20\end{array}$ & $\begin{array}{r}-160,00 \\
-51,60\end{array}$ & $\begin{array}{l}\mathrm{M}_{\mathrm{C}} \\
3,0\end{array}$ & este trabajo \\
\hline 6 & $16 / 10 / 2008$ & $09: 26: 43.0$ & $-33,04$ & $-68,89$ & 17,3 & $\pm 8,0$ & $\begin{array}{l}355,87 \\
250,80\end{array}$ & $\begin{array}{l}72,61 \\
50,30\end{array}$ & $\begin{array}{r}42,00 \\
157,10\end{array}$ & $\begin{array}{l}\mathrm{M}_{\mathrm{C}} \\
2,4\end{array}$ & este trabajo \\
\hline
\end{tabular}

Se utilizó $\mathrm{M}_{\mathrm{c}}$ (Magnitud Coda, cuando no se contó con datos suficientes para realizar inversión de formas de onda y obtener M Magnitud Momento).

sísmicas, se interpreta como un pliegue asociado al movimiento de esta falla (Fig. 8). A partir de la integración de los datos obtenidos en superficie con las interpretaciones de los datos de subsuelo, se modeló esta estructura con un despegue basal ubicado a 10-11 km de profundidad. La falla presentaría un sector norte de rumbo NNW, con fuerte inclinación al oeste, y un sector sur de rumbo norte-sur, con menor inclinación al oeste. Este cambio de geometría entre ambos sectores es interpretado como producto de la reactivación de una falla extensional triásica de rumbo NNW, en el sector norte (Fig. 9).

La propagación de la falla Maure hacia el este genera un punto ciego ubicado por debajo del sector oeste de la ciudad de Mendoza, desde el cual se propagan fallas retrovergentes responsables del levantamiento del sistema Cerro de la Gloria. A partir del modelado cinemático se infiere que el crecimiento de este sistema estructural se habría producido desde el Plioceno Tardío, durante la depositación de los estratos de la Formación Mogotes, y habría sido activo hasta el presente.

\subsection{Análisis de mecanismos focales}

La figura 10 y la tabla 2 muestran las soluciones de los mecanismos focales resueltos para la zona estudiada y áreas adyacentes. Considerando las incertidumbres en la profundidad del foco sísmico, solo los mecanismos 1 y 6 pueden asociarse a las estructuras presentadas en este trabajo. El mecanismo focal 1, resuelto por Alvarado et al. (2005) con inversión de forma de onda, tiene solución inversa. El plano más probable es el 181, 19, 77 (rumbo/manteo/deslizamiento) el cual podría interpretarse como asociado al despegue de la falla Maure. Los mecanismos focales 2, 3, 5 (asociados a estructuras normales) y el 4 se asocian a niveles más profundos, no analizados en este trabajo. Los mecanismos 2 y 3 se encuentran dentro de una franja de orientación NW-SE de mecanismos normales interpretada por Nacif et al. (2013) como el producto de la existencia de algún tipo de esfuerzo de relajación temporal que permite que señales de régimen extensional aparezcan dentro de un ambiente compresivo.

En el caso del mecanismo focal 5, si bien el error en la determinación permitiría ubicarlo en profundidades correspondientes al modelo estructural presentado (profundidad 10,1 $\pm 8,5$ ), no se puede relacionar con ninguna estructura identificada en la región, por lo tanto, se infiere que estaría asociado a estructuras más profundas como la de los sismos 2 y 3 .

El plano nodal del mecanismo focal 6 que tiene una profundidad de $17 \pm 8 \mathrm{~km}$, estaría relacionado con la falla transcurrente de los Pozos. Si esto es correc- 

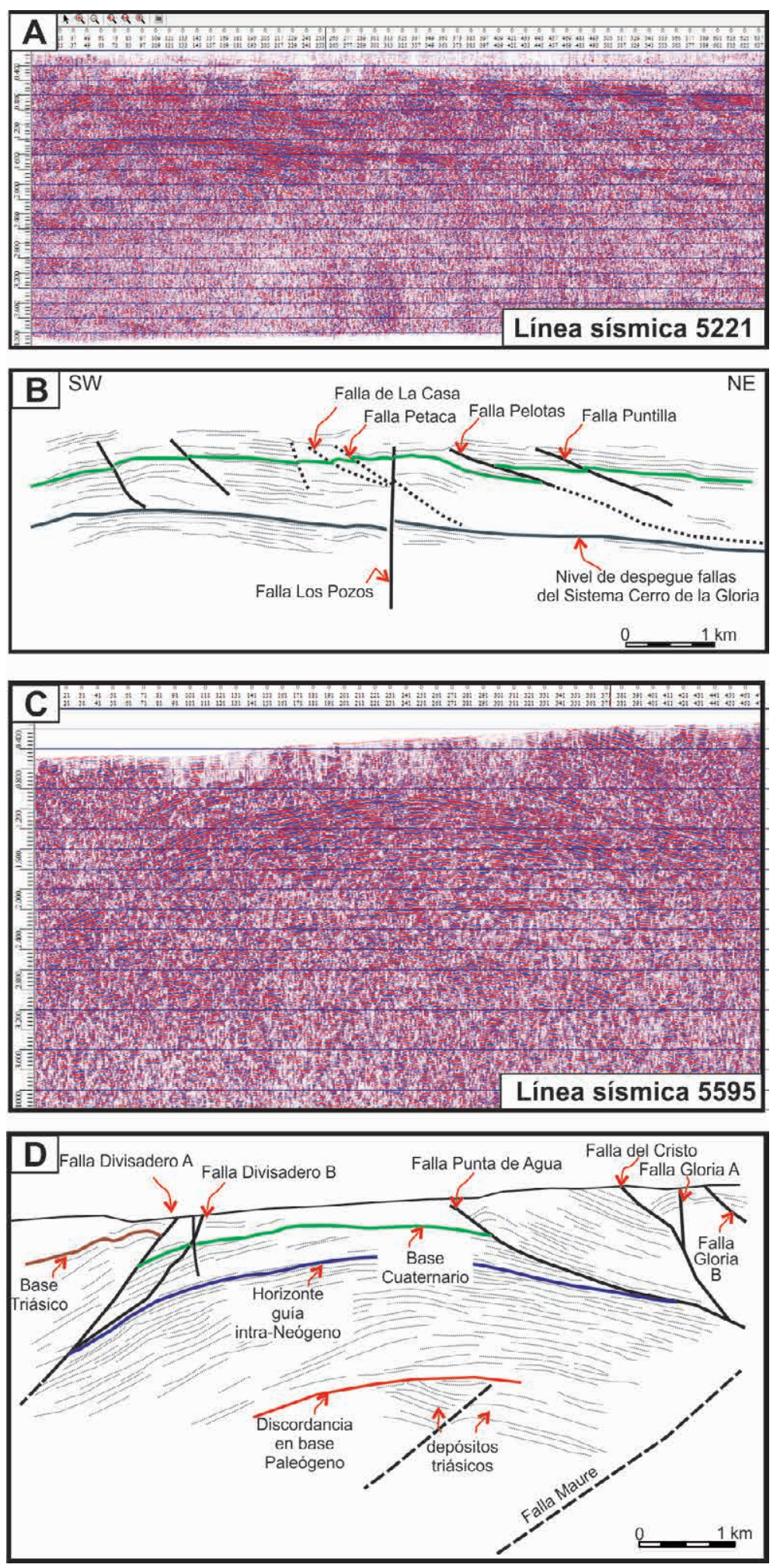

FIG. 8. Perfiles interpretados de las líneas sísmicas 5.221 y 5.595 graficadas en la figura 1. 


\section{Perfil estructural balanceado a los $32^{\circ} 53^{\prime} 50^{\prime \prime} \mathrm{S}$}

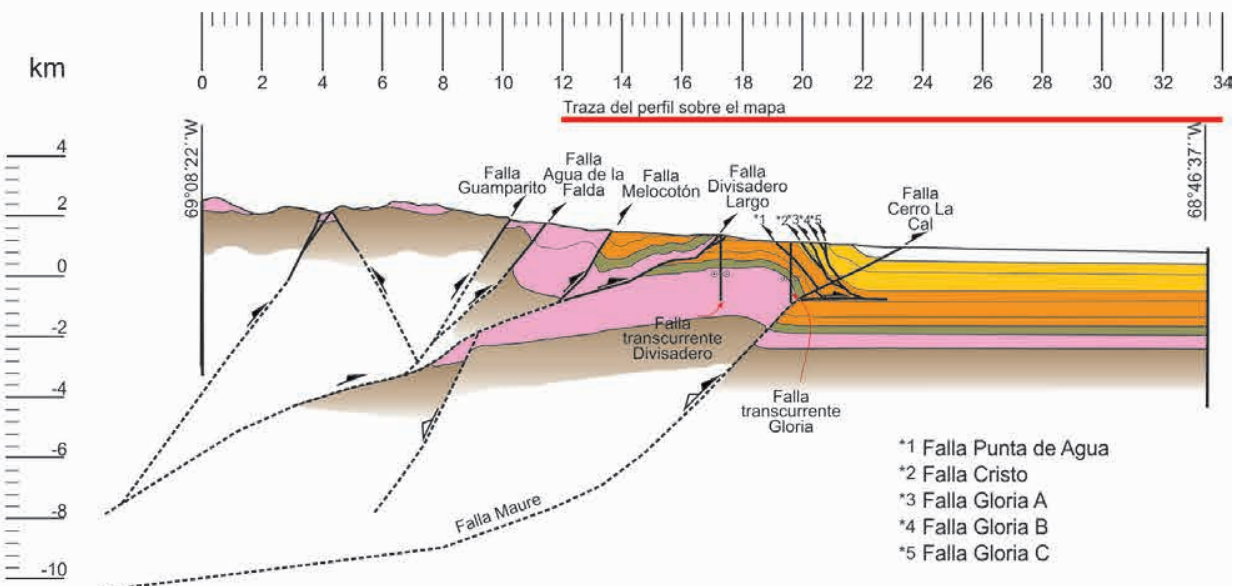

Perfil estructural balanceado a los $32^{\circ} 55^{\prime} 50^{\prime \prime} \mathrm{S}$
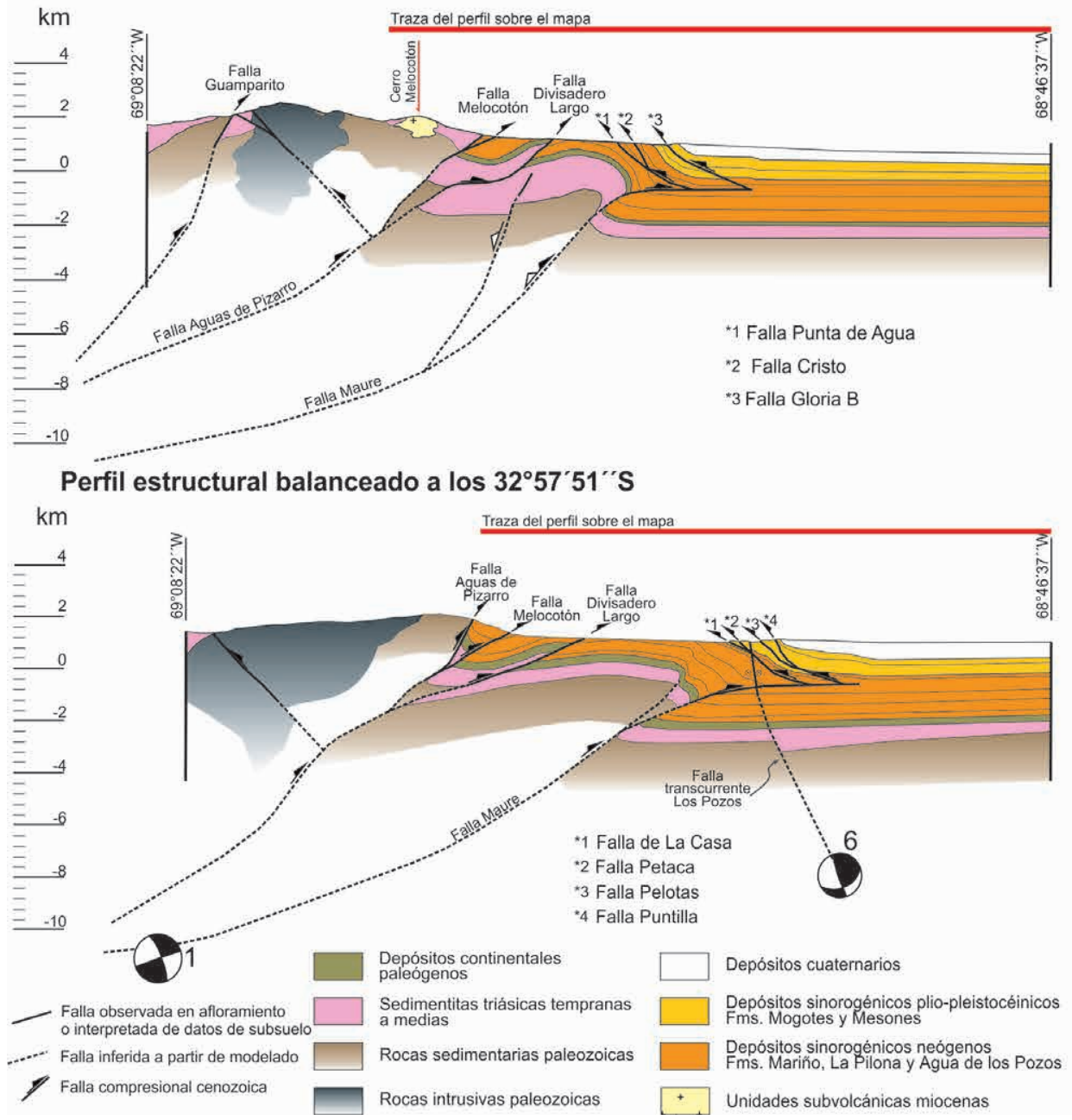

FIG. 9. Perfiles balanceados obtenidos con datos de campo y líneas sísmicas. 


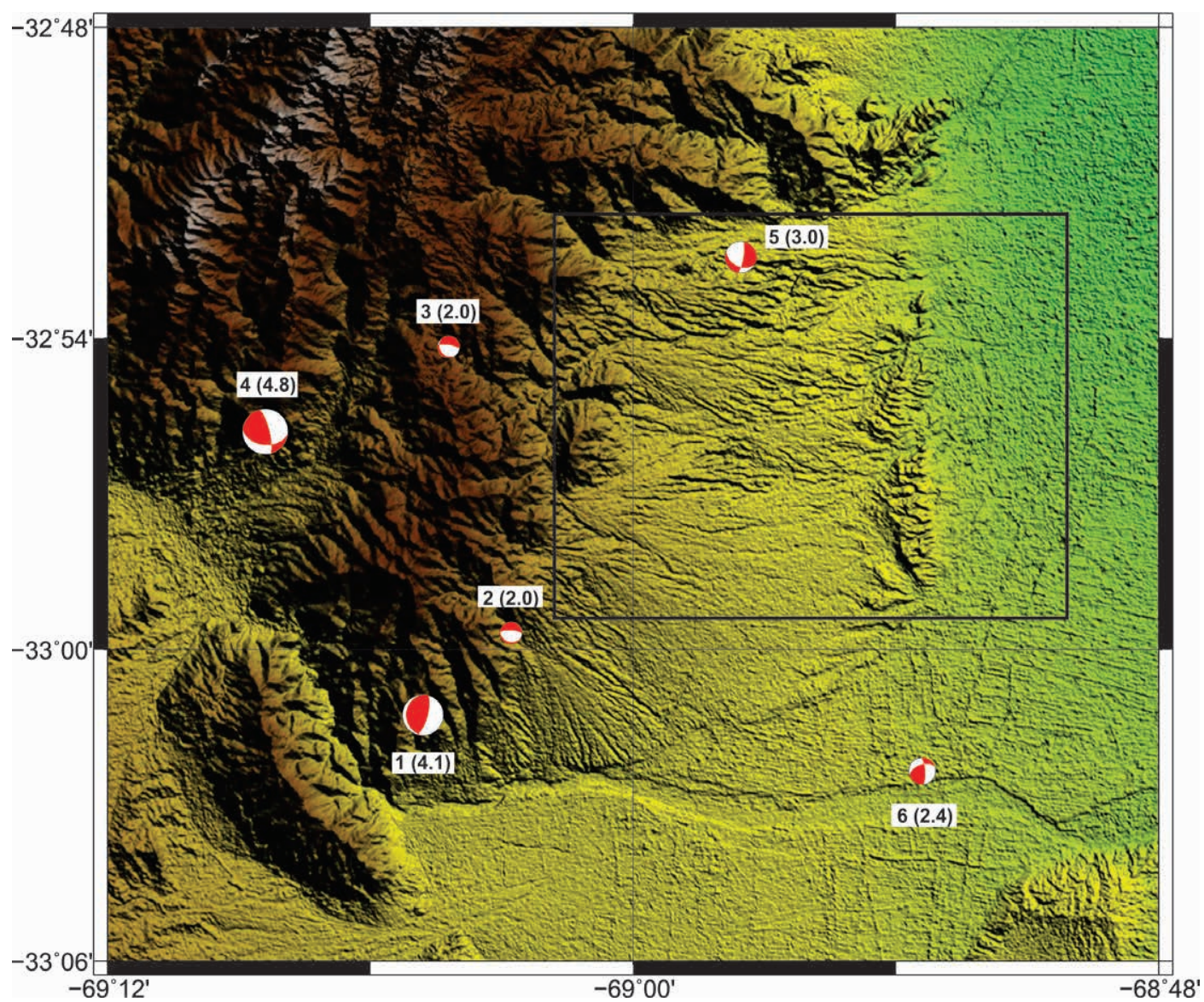

FIG. 10. Soluciones de mecanismos focales de doble cupla para la región de estudio y áreas adyacentes. Cuadrante compresional: color rojo.

to, dicha falla alcanzaría profundidades mayores a los $9 \mathrm{~km}$ de profundidad, lo que es consistente con la interpretación de las fallas transcurrentes como estructuras antiguas reactivadas. Si bien el número de mecanismos focales presentados en este estudio no tiene una representación estadística significativa $(\mathrm{N}=6)$, los resultados muestran una amplia diversidad. Las soluciones que no se contemplan en el modelo aquí presentado deberían ser tenidas en cuenta en futuros estudios de riesgo sísmico.

\section{Discusión y conclusiones}

Las interrogantes en cuanto a la evolución espacio-temporal de las fallas del frente orogénico a la latitud de la ciudad de Mendoza se remontan a casi más de 100 años. Keidel (1907) asume que la actividad de las fallas pedemontanas debió comen- zar en el Plioceno, aunque habría aumentado en el Pleistoceno. Loos (1926) asegura que dicha actividad habría continuado hasta la actualidad. Los perfiles geoeléctricos realizados en los alrededores del cerro de la Gloria evidencian la deformación de los niveles pliocenos de la Formación Mogotes (Perelló, 1991) que se observan cas verticales en las cercanías del cerro de la Gloria.

Los estudios realizados en los últimos 20 años demuestran que los cuatro principales sistemas de fallas del frente orogénico andino a estas latitudes poseen evidencias de actividad cuaternaria. El sistema de fallas Cerro de La Gloria y Cerro el Cristo afectan conglomerados pleistocenos y una secuencia de limos de posible edad holocénica (Milana y Zambrano, 1996). La falla Divisadero Largo levanta rocas triásicas de la Formación Potrerillos por encima de sedimentos cuaternarios (Rodríguez y Barton, 
1990), mientras que la falla Melocotón desplaza limos cuaternarios que contienen gastrópodos datados en 38.000 años (INPRES, 1995). La falla Cerro La Cal afecta niveles aluviales pleistocénicos y depósitos modernos (INPRES, 1995) y ha sido vinculada a una deformación histórica para dos tramos de la falla (Mignorance, 2006; Schmidt et al., 2011; Salomon et al., 2013).

El modelo cinemático para estas estructuras desarrollado en el presente trabajo corresponde a corrimientos imbricados con despegue profundo ubicado aproximadamente a los 10-11 km de profundidad en basamento paleozoico y conectado a partir de la presencia de una importante falla ciega, denominada Maure, cuya existencia es corroborada con datos de subsuelo. Los sismos compresivos registrados a profundidades $\leq 10 \mathrm{~km}$ pueden asociarse al movimiento de este despegue superficial. Dicha estructura se conecta con las estructuras más someras, observadas en superficie, ya sea a partir de corrimientos asociados, como las fallas Divisadero y Melocotón o como retrocorrimientos someros generados a partir del avance de una cuña orogénica formada por la falla Maure hacia el antepaís. Un modelo similar, en el cual una falla profunda de basamento transfiere rechazo hacia la superficie a partir de la generación de una zona triangular en el piedemonte, ha sido propuesto por Vergés et al. (2007) para el sector ubicado $30 \mathrm{~km}$ al norte del estudiado en este trabajo. Estos autores proponen la existencia de un despegue profundo, por debajo de los $10 \mathrm{~km}$ de profundidad, desde el cual se desprende un corrimiento vergente al este. Este corrimiento transfiere rechazo en la interfase rocas cristalinas proterozoicas-calizas cámbricas hacia corrimientos de piel fina retrovergentes, generando de esta manera una zona triangular similar a la propuesta en este trabajo. Sin embargo, en el área estudiada, los datos de subsuelo muestran que las fallas retrovergentes se enraízan en un despegue más somero, ubicado dentro de los depósitos sinorogénicos neógenos y las rocas paleozoicas se encuentran afectadas por la falla Maure.

Si bien, la actividad sísmica actual de este sector del piedemonte mendocino es moderada con sismos superficiales que no superan M 4, los rasgos morfométricos de las fallas estudiadas permiten estimar magnitudes máximas probables mucho más elevadas. En este contexto, la peligrosidad sísmica del piedemonte mendocino teniendo en cuenta la falla ciega Maure relativamente superficial implica escenarios peores a los señalados por autores previos. Se estima una magnitud máxima probable $\mathrm{Mw}=6,6$ para esta potencial fuente sismogénica asociada a sismos superficiales. Las fallas corticales someras suelen presentar una baja recurrencia del orden de miles a decenas de miles de años; sin embargo, se han registrado rupturas superficiales durante sismos prehistóricos con un rechazo vertical cosísmico superior a $1 \mathrm{~m}$ en el sector estudiado. Esto demuestra una capacidad sísmica mayor a la sugerida en catálogos sísmicos (Costa et al., 2006).

Al sistema compresivo principal se sobreponen fallamientos transcurrentes que no habían sido mapeados con anterioridad y cuya sismicidad no ha sido tenido en cuenta hasta este trabajo. El mecanismo focal del sismo del día 16/10/2008 se asocia al movimiento de estos sistemas.

Los mecanismos focales permiten reconocer la orientación, vergencia y tipo de movimiento de las fallas que actúan como fuentes sismogénicas. Estos datos son fundamentales para evaluar qué tipo de estructuras son responsables de la actividad sísmica. Los mecanismos focales de Alvarado et al. (2005) y el del sismo del día 16/10/2008 son mecanismos inversos con pequeñas componentes de rumbo, lo cual permite inferir la existencia de acortamientos corticales debidos a un régimen regional de esfuerzos compresivos asociados a las fallas analizadas en este trabajo.

A partir de este trabajo surge la necesidad de realizar un experimento sismológico local para evaluar en detalle la sismicidad. Es de vital importancia la precisa localización de la sismicidad para luego realizar una confiable asociación con las estructuras de la zona de estudio. Sin embargo, los resultados obtenidos hasta el momento son de particular interés para la peligrosidad sísmica de la ciudad de Mendoza que ha experimentado una considerable expansión urbana en los últimos años.

\section{Agradecimientos}

Este trabajo se ha realizado en el marco del proyecto PIP 112201101-00484 (2012-2014, Res. 1672). Se agradece a INPRES por los datos sismológicos. Los autores desean agradecer a los revisores C. Costa y E. Godoy por sus útiles comentarios que han contribuido a mejorar este manuscrito. 


\section{Referencias}

Ahumada, E.A. 2006. Neotectónica del frente orogénico andino entre $32^{\circ} 08^{\prime}$ S $-32^{\circ} 19^{\prime}$ 'S (provincias de Mendoza y San Juan). Tesis Doctoral (Inédito), Universidad Nacional de San Luis: 163 p.

Ahumada, E.A.; Costa, C.H.; Gardini, C.E.; Diederick, H. 2006. La estructura del extremo sur de la sierra de las Peñas-Las Higueras, precordillera de Mendoza. Revista de la Asociación Geológica Argentina, Publicación Especial 9, Serie D: 11-17.

Ahumada, E.A.; Costa, C.H. 2009. Deformación cuaternaria en la culminación norte del corrimiento las Peñas, frente orogénico andino, Precordillera Argentina. In Congreso Geológico Chileno, No. 12, CD-Rom: 4 p. Santiago.

Aki, K.; Richards, P.G. 1980. Quantitative Seismology, Theory and Methods. Freeman and Company 1-2:932p. San Francisco.

Alvarado, P.; Beck, S.; Zandt, G.; Araujo, M.; Triep, E. 2005. Crustal deformation in the south central Andes backarc terranes as viewed from regional broadband seismic waveform modeling. Geophysical Journal International 60 (2): 580-598.

Ave Lallemant, G. 1892. Observaciones sobre el mapa del Departamento de Las Heras, provincia de Mendoza. Anales del Museo de La Plata, Sección Geológica y Mineralógica 1: 5-20.

Bastías, H. 1986. Fallamiento Cuaternario en la Región Sismotectónica de Precordillera. Tesis Doctoral(Inédito), Facultad de Ciencias Exactas, Físicas y Naturales, Universidad Nacional de San Juan: 147 p. Argentina.

Bastías, H.; Tello, G.E.; Perucca, J.L.; Paredes, J.D. 1993. Peligro sísmico y geotectónica. In Congreso Geológico Argentino, No. 12 y Congreso de Exploración de Hidrocarburos, No. 2. Geología y Recursos Naturales de Mendoza (Ramos, V.A.; editor), Relatorio 6-1: 645-658. Mendoza.

Bastías, H.; Weidmann, N.; Pérez, A. 1984. Dos zonas de fallamiento Plio-Cuaternario en la Precordillera de San Juan. In Congreso Geológico Argentino, No. 9, Actas 2: 329-341. San Carlos de Bariloche.

Bastías, H.; Uliarte, E.; Paredes, J.; Sánchez, A.; Bastías, J.; Ruzycki, L.; Perucca, L. 1990. Neotectónica de la provincia de San Juan. In Congreso Geológico Argentino, No. 11. Relatorio de Geología y Recursos Naturales de la provincia de San Juan: 228-245. San Juan.

Bodenbender, G. 1897. El suelo y las vertientes de la ciudad de Mendoza y sus alrededores. Boletín de la Academia Nacional de Ciencias de Córdoba 15: 425-486.
Bodenbender, G. 1902. Contribución al conocimiento de la Precordillera y de la Sierras Centrales de la República Argentina. Boletín Academia Nacional de Ciencias de Córdoba 18: 203-264.

Brooks, B.A.; Sandvo, L.E.; Ross, A. 2000. Fold style inversion: placing probabilistic constraints on the predicted shape of of blind thrust fault. Journal of Geophysical Research 105: 13281-13302.

Brooks, B.A.; Bevis, M.; Smalley, R.; Kendrick, E.; Manceda, R.; Lauria, E.; Maturana, R.; Araujo, M. 2003. Crustal motion in the Southern Andes $\left(26^{\circ}\right.$ $\left.36^{\circ} \mathrm{S}\right)$ : Do the Andes behave like a microplate? Geochemistry, Geophysics, Geosystems 4 (10). doi: 10.1029/2003GC000505.

Burbank, D.W.; Anderson, R.S. 2001. Tectonic Geomorphology. Backwell Science: 274 p. Oxford.

Chiaramonte, L.; Ramos, V.A.; Araujo, M. 2000. Estructura y sismotectónica del anticlinal de Barrancas, cuenca cuyana, provincia de Mendoza. Revista de la Asociación Geológica Argentina 55 (4): 309-336.

Costa, C.H.; Machette, M.N.; Dart, R.L.; Bastías, H.E.; Paredes, J.D.; Perucca, L.P.; Tello, G.E.; Haller, K.M. 2000a. Map and Database of Quaternary Faults and Folds in Argentina. United States Geological Survey (USGS), Open-File Report 00-0108: 81 p.

Costa, C.H.; Gardini, C.E.; Diederix, H.; Cortés, J.M. 2000b. The Andean orogenic front at Sierra de Las Peñas-Las Higueras, Mendoza, Argentina. Journal of South America Earth Science 13 (3): 287-292.

Costa, C.H.;Audemard, F.A.; Bezerra, F.H.; Lavenu, R.A.; Machette, M.N.; París, G. 2006. An overview of the main quaternary deformation of South America. Revista de la Asociación Geológica Argentina 61 (4): 461-479.

Cortés, J.M.; Vinciguerra, P.; Yamín, M.; Pasini, M.M. 1999. Tectónica cuaternaria de la Región Andina del Nuevo Cuyo (28 $\left.-38^{\circ} \mathrm{LS}\right)$. In Geología Argentina (Caminos, R.; editor). Servicio Geológico Minero Argentino, Subsecretaría de Minería, Anales 29 (24): 760-778. Buenos Aires.

Cortés, J.M.; Casa, A.; Pasini, M.; Yamín, M.; Terrizano, C. 2006. Fajas oblicuas de deformación neotectónica en Precordillera y Cordillera Frontal (31 $\left.30-33^{\circ} 30^{\prime} \mathrm{LS}\right)$ : Controles paleotectónicos. Revista de la Asociación Geológica Argentina 61 (4): 639-646.

Folguera, A.; Etcheverría, M.; Pazos, P.; Giambiagi, L.B.; Cortés, J.M.; Fauqué, L.; Fusari, C.; Rodríguez, M.F. 2004. Descripción de la Hoja Geológica Potrerillos (1:100.000). Subsecretaría de Minería de la Nación, Dirección Nacional del Servicio Geológico: 262 p. 
Fossa Mancini, E. 1939. Vistas aéreas, fallas activas y temblores mendocinos. Boletín de informaciones petroleras 178: 45-77.

Gapais, D.; Cobbold, P.; Bourgeois, O.; Rouby, D.; Urreiztieta, M. 2000. Tectonic significance of faultslip data. Journal of Structural Geology 22: 881-888.

Giambiagi, L.B.; Moreiras, S.M. 2009. Informe final: Terreno construcción Escuela Barrio Sol y Sierra. Municipalidad de Godoy Cruz: 6 p. Mendoza.

Giambiagi, L.; Mescua, J.; Bechis, F.; Martínez, A.; Folguera, A. 2011. Pre-Andean deformation of the Precordillera southern sector, Southern Central Andes. Geosphere 7 (1): 1-21.

González, M.A.; González Díaz, E.F.; Sepúlveda, E.; Regairaz, M.C.; Costa, C.; Cisneros, H.; Bea, S.; Gardini, C.; Pérez, I.; Pérez, M. 2002. Carta de peligrosidad geológica 3369-II, Mendoza. Provincias de Mendoza y San Juan. Servicio Geológico Minero Argentino, Subsecretaría de Minería de la Nación, Boletín 324: 178 p.

Hardebeck, J.L.; Shearer, P.M. 2002. A new method for determining first-motion focal mechanisms. Bulletin of the Seismological Society of America 92 (6): 2264-2276.

Hardebeck, J.L.; Shearer, P.M. 2003. Using S/P amplitude ratios to constrain the focal mechanisms of small earthquakes. Bulletin of the Seismological Society of America 93 (6): 2434-2444.

Harrington, H. 1944. El sismo de San Juan del 15 de enero de 1944. Corporación para la Promoción del Intercambio S.A.: 79 p.

Havskov, J.; Ottemoller, L. 1999. SEISAN earthquake analysis software. Seismological Research Letters 70 (5): 532-534

Havskov, J.; Ottemoller, L. 2002. SEISAN earthquake analysis and SEISNET network automation software, International Handbook of Earthquake and Engineering Seismology 81B: 15-16.

INPRES (Instituto Nacional de Prevención Sísmica). 1985. El terremoto de Mendoza Argentina del 26 de enero de 1985. Informe general: 139 p. San Juan.

INPRES (Instituto Nacional de Prevención Sísmica). 1995. Microzonificación sísmica del Gran Mendoza. Instituto Nacional de Prevención Sísmica. Resumen Ejecutivo. Publicación Técnica, No. 19: 269 p. San Juan.

Irigoyen, M.; Brown, R.; Buchan, K. 1999. Cronología neogénica de los depósitos sinorogénicos aflorantes en la región de Cachueta/Tupungato, Norte de Mendoza. In Congreso Geológico Argentino, No. 16, Actas 1: 63-64. La Plata.
Irigoyen, M.V.; Buchan, K.L.; Brown, R.L. 2000. Magnetostratigraphy of Neogene Andean forelandbasin strata, lat $33^{\circ} \mathrm{S}$, Mendoza Province, Argentina. Geological Society of America, Bulletin 112 (6): 803-816.

Keidel, H. 1907. Über den Bau der Argentinischen Andes Sitzungsberichte der kaiserlichen Akademie der Wissenshaften in Wein, Mathematisch Naturwissenschftliche Klasse. Tomo 116 (1): 649-674.

Keidel, J. 1910. Informe sobre los trabajos efectuados por la sección geología de la División de Minas. Geología e Hidrología en los años 1906, 1907 y 1908. Memoria año 1908. Anales del Ministerio de Agricultura, Sección Geología, Mineralogía y Minas. Tomo 5 (2): 26-77.

Kendrick, E.; Brooks, B.A.; Bevis, M.; Smalley, R.; Lauria, E.; Araujo, M.; Parra, H. 2006. Active Orogeny of the South-Central Andes studied with GPS Geodesy. Revista de la Asociación Geológica Argentina 61 (4): 555-566.

Kurtz, F. 1897. Enumeración de las plantas recogidas por Bondebender en la Precordillera de Mendoza (octubre de 1896). Bolentín de la Academia Nacional de Ciencias de Córdoba. Tomo 15: 502-522.

Liener, B.R.; Havskov, J. 1995. A Computer Program for Locating Earthquakes Both Locally and Globally. Seismological Research Letters 66 (5): 26-36.

Loos, P. 1907. Estudios de sismología. Los movimientos sísmicos de Mendoza. Anales del Ministerio de Agricultura, Sección Geología, Mineralogía y Minas, Tomo 3 (1): 1-38.

Loos, P. 1926. Los terremotos del 17 de diciembre de 1920 en Costa de Araujo, Lavalle, La Central, Tres Porteñas. Contribuciones Geofísicas del Observatorio Astronómico de la Universidad de La Plata. Tomo 1 (2): 129-158.

Marrett, R.; Allmendinger, R.W. 1990. Kinematic análisis of fault-slip data. Journal of Structural Geology 12: 973-986.

Meigs, A.J.; Nabelek, J. 2010. Crustal-scale pure shear foreland deformation of western Argentina. Geophysical Research Letters 37 (5): 11.304 p. doi: 10.1029/2010GL043220

Milana, J.P.; Zambrano, J.J. 1996. La Cerrillada Pedemontana Mendocina: un sistema geológico retrocorrido en vías de desarrollo. Revista de la Asociación Geológica Argentina 51 (4): 289-303.

Mignorance, F. 2006. Morfometría de la falla histórica de la zona de falla La Cal. Revista de la Asociación Geológica Argentina 61 (4): 620-638. 
Moreiras, S.M. 2004. Zonificación de peligrosidad y de riesgo de procesos de remoción en masa en el valle del río Mendoza. Provincia de Mendoza. Tesis doctoral (Inédito). Universidad Nacional San Juan: 274 p

Moreiras, S.M. 2010. Riesgo geológico del piedemonte mendocino. Amenazas naturales y antrópicas en el piedemonte de la precordillera de San Juan y Mendoza (Alberto, I.; Vich, J.; editores). Propuestas para su mitigación y control. Editorial Zeta: 75-90.

Morey, F. 1938. Los temblores de tierra. Mendoza Sísmica. Editorial D'Accurzio: 102 p. Mendoza.

Nacif, S.V. 2006. Sismicidad cortical de pequeñas magnitudes $(\mathrm{M}<3.5)$ en el terreno de Cuyania y zona de borde con Chilenia obtenidas de red temporal de banda ancha. Tesis de grado de Licenciatura en Geofísica (Inédito), Universidad Nacional de San Juan: 109 p.

Nacif, S.; Triep, E.; Furlani, R.; Spagnotto, S. 2013. Stripe of normal mechanisms for crustal earthquakes with $\mathrm{M} \leq 3.5$ flanking the western side of the thrust front zone in the Andes backarc. Natural Science 5: 18-26. doi: 10.4236/ns.2013.58A1003

NEIC (National Earthquake International Center). 2014. United States Geological Survey Web site.

Olgiati, S. 2002. El anticlinal de Borbollón y la estructura del frente precordillerano de Mendoza. Tesis licenciatura (Inédito), Universidad de Buenos Aires: $107 \mathrm{p}$.

Olgiati, S.; Ramos, V.A. 2003. Neotectónica Cuaternaria en el Anticlinal Borbollón, provincia de MendozaArgentina. In Congreso Geológico Chileno, No. 10, CD Rom: 11 p. Concepción.

Perelló, C.R. 1991. Estudio Geofísico del Cerro de la Gloria, provincia de Mendoza. Tesis. Universidad Nacional de San Juan: 94 p.

Perucca, L.P.; Moreiras, S.M. 2010. Seismic and volcanic hazard in Argentina. In Natural Hazards and HumanExacerbated Disasters in Latin-America. Special volume of geomorphology: Developments in Earth surface processes. Elsevier: 267-297.

Polanski, J. 1953. Supuestos englazamientos de la llanura pedemontana de Mendoza. Revista de la Asociación Geológica Argentina 8 (4): 195-213.

Polanski, J. 1958. El bloque varíscico de la Cordillera Frontal de Mendoza. Asociación Geológica Argentina, Revista 12 (3): 165-193.

Polanski, J. 1963. Estratigrafía, neotectónica y geomorfología del Pleistoceno pedemontano entre los ríos Diamante y Mendoza (provincia de Mendoza). Revista de la Asociación Geológica Argentina 17 (3-4): 127-349.
Reasenberg, P.A.; Oppenheimer, D. 1985. FPFIT, FPPLOT, and FPPAGE: Fortran computer programs for calculating and displaying earthquake fault-plane solutions. United States Geological Survey Open-File Report: 85-739.

Reigaraz, A.C.; Zambrano, J.J. 1991. Unidades morfoestructurales y fenómenos neotectónicos en El norte de la província de Mendoza (Andes Centrales argentinos entre $32^{\circ}$ y $34^{\circ}$ de latitud sur). Bamberger Geografische Schriften 11: 1-21.

Rodríguez, E.J.; Barton, M. 1990. Geología del pie de monte del oeste de la ciudad de Mendoza. In Congreso Geológico Argentino, No. 10, Actas 1: 460-463. Tucumán.

Salomon, E.; Schmidt, S.; Hetzel, R.; Mingorance, F.; Hampel, A. 2013. Repeated folding during late holocene earthquakes on the La Cal thrust fault near Mendoza City (Argentina). Bulletin of the Seismological Society of America 103 (2A): 936-949.

Sepúlveda, E.; López, H. 1998. Descripción geológica de la Hoja 3369-II Mendoza, provincia de Mendoza. Servicio Geológico Minero Argentino (SEGEMAR), Boletín 252: p. 75. Buenos Aires.

Siame, L.; Bellier, O.; Sebrier, M. 2006. Active tectonics in the Argentine Precordillera and western Sierras Pampeanas. Revista de la Asociación Geológica Argentina 61 (4): 604-619.

Snoke, J.A.; Munsey, J.W.; Teague, A.C.; Bollinger, G.A. 1984. A program for focal mechanism determination by combined use of polarity and SV-P amplitude ratio data. Earthquakes Notes 55: 3-15.

Schmidt, S.; Hetzel, R.; Mingorance, F.; Ramos, V.A. 2011. Coseismic displacements and Holocene slip rates for two active thrust faults at the mountain front of the Andean Precordillera $\left(\sim 33^{\circ} \mathrm{S}\right)$. Tectonics 30 (TC5011). doi: 10.1029/2011TC002932.

Slemmons, D.B. 1977. Strate of the art for assessing earthquake hazards in the United States: Report 6, Faults and Earthquake Magnitude for United States Army Engineer Waterways Experiment Station, Vicksburg, Mississippi, Miscellaneous Paper S-73-1: 129 p.

Stappenbeck, R. 1910. La Precordillera de San Juan y Mendoza, Anales del Ministerio de Agricultura. Sección Geología 4 (3): 3-187.

Stappenbeck, R. 1913. El agua subterránea al pie de la Cordillera Mendocina y Sanjuanina. Anales del Ministerio de Agricultura, Sección Geología, Mineralogía y Minas 7 (5): 69 p.

Twiss, R.J.; Unruh, J.R. 1998. Analysis of fault slip inversions: Do they constrain stress or strain rate? 
Journal of Geophysical Research B: Solid Earth 103 (6): 12205-12222.

Vergés, J.; Ramos, V.A.; Meigs, A.; Cristallini, E.; Bettini, F.H.; Cortés, J.M. 2007. Crustal wedging triggering recent deformation in the Andean thrust front between $31^{\circ} \mathrm{S}$ and $33^{\circ} \mathrm{S}$ Sierras Pampeanas-Precordillera interaction. Journal of Geophysical Research 112 (3) (B03S15). doi: 10.1029/2006JB004287.

Vich, A.I.; Pedriani, A. 1992014.3. Programa de Investigación y Desarrollo: manejo Ecológico del Piedemonte. Mendoza. Ministerio de Medio Ambiente Urbanismo y Vivienda-Centro Regional de Investigaciones Científicas y Tecnológicas (CRICYT),
Unidad de manejo Ecológico de Cuencas, Fase I: 154 p. Mendoza.

Volponi, F.; Quiroga, M.; Robles, A. 1978. El terremoto de Caucete del 23 de noviembre de 1977. Instituto Sismológico Zonda. Universidad Nacional de San Juan: 81 p.

Wallace, B. 1986. New horizons in digital signal processing. Noise and Vibration Worldwide 17 (8): 234-237.

Wells, D.L.; Coppersmith, K.J. 1984. New empirical relationships among magnitude, rupture length, rupture width, rupture area, and surface displacement. Bulletin of Seismological Society of America 84 (4): 974-1002.

Manuscript received: November 07, 2012; revised/accepted: October 28, 2013; available online: November 04, 2013. 